\title{
Early mismatch between cancer cells and their tumour microenvironment blocks long term growth
}

Anna Perdrix Rosell ${ }^{1,2,3}$, Oscar Maiques ${ }^{2,3}$, Probir Chakravarty ${ }^{4}$, Luigi Ombrato ${ }^{1,5}$, Victoria Sanz Moreno ${ }^{2,3 \#} \&$ Ilaria Malanchi ${ }^{1 \#}$

${ }^{1}$ Tumour Host Interaction Laboratory, The Francis Crick Institute, 1 Midland Road,NW1 1AT London, UK.

2 Sanz-Moreno Lab, Centre for Tumour Microenvironment, Barts Cancer Institute, Queen Mary University of London, Charterhouse Square, London EC1M 6BQ, UK.

${ }^{3}$ Randall Division of Cell and Molecular Biophysics, King's College London, New Hunt's House, Guy's Campus, London SE1 1UL, UK.

${ }^{4}$ Bioinformatics and Biostatistics Unit, The Francis Crick Institute, 1 Midland Road,NW1 1AT London, UK.

${ }^{5}$ Current address: Ombrato Lab, Centre for Tumour Microenvironment, Barts Cancer Institute, Queen Mary University of London, Charterhouse Square, London EC1M 6BQ, UK.

\# correspondence to v.sanz-moreno@qmul.ac.uk and llaria.malanchi@crick.ac.uk.

Running title: Tailored TME is needed for cancer growth

\section{Abstract}

Cancer cells thrive embedded in a fine-tuned cellular and extracellular environment or tumour microenvironment (TME). There is the general understanding of a co-evolution between cancer cells and their surrounding TME, suggesting a functional connection between cancer cells characteristics and the perturbations induced in their surrounding tissue. However, this functional connection has never been formally proven with a dedicated experimental setting. Here we provide experimental evidence that when aggressive cancer cells are forced to initially establish an alternative TME, this will evolve to impair long term in vivo cancer growth. Using a combination of in vivo transcriptomics, protein arrays and in vitro co-cultures, we found that this alternative TME culminates in the infiltration of macrophages with STAT1 ${ }^{\text {high }}$ activity. Despite lacking canonical M1 markers, these macrophages show strong anti-tumoural functions which reduce long-term tumour growth. Importantly, gene signatures of stromal cell, the major cellular component in the alternative TME as well as the alternative activated macrophages show striking prognostic power, correlating with less aggressive human breast cancers. 


\section{Introduction}

38 Cancer cells have the ability to generate a special cellular and extracellular environment to support their growth, which is collectively named tumour microenvironment (TME). This local ecosystem comprises an acellular part, characterized by a dense network of extracellular matrix (ECM) proteins, as well as a variety of cellular components (Georgouli et al. 2019). For instance, cancer-associated fibroblasts (CAFs) are one of the dominant components of the TME and master secretors of ECM proteins and play a key role in tumour progression, tumour invasion and metastasis (Östman \& Augsten 2009; Sanz-Moreno et al. 2011; Malanchi et al. 2011). Moreover, a multifaceted array of innate immune-cells, such as monocytes, macrophages and neutrophils, promotes tumorigenesis and metastatic dissemination (Coussens \& Werb 2002; Ogura et al. 2017; Wculek \& Malanchi 2015). However, macrophages, which have a pivotal role in orchestrating cancer related inflammation, can have a dual supportive or inhibitory role in the cancer context(Mantovani et al. 2017). Importantly, the type of intra-tumoral immune infiltration in different cancer contexts is determined by the properties of the TME, for instance CAFs can contribute to the recruitment of immunosuppressive cells: tumour-associated macrophages (TAMs) or regulatory T lymphocytes (Costa et al. 2018).

An essential aspect of aggressive cancer cells is their ability to change their cytoskeleton to grow, invade, metastasize and evade the immune system. A key regulator of the cytoskeleton is Rho-associated coiled-coil containing protein kinase (ROCK) which increases Myosin II activity (Vicente-Manzanares et al. 2009) and cancer invasion. Aside from its well described role in metastasis (Rodriguez-Hernandez et al. 2016), ROCK has been shown to promote intrinsic tumorigenic ability of tumours of mesenchymal origin such as melanoma (Rodriguez-Hernandez et al. 2020; Kümper et al. 2016; Wong et al. 2020). Importantly, the tumorigenic potential of cancer cells is coupled to their extrinsic ability to promote a tumour supporting TME (del Pozo Martin et al. 2015). Indeed, the fact that Myosin II activity in melanoma cells results in a different secretory profile which alters the properties of TME components (Georgouli et al. 2019; Orgaz et al. 2020), advocate for the idea that the activation of pathways supporting cancer cells tumorigenic potential is directly linked to their ability to create their optimal cellular ecosystem within that tissue.

The fact that different tumour types have different cellular composition in their TME (Wagner et al. 2019), rises the intriguing possibility that a specific match between the intrinsic characteristics of cancer cells and the kind of TME established is required to support their growth. In this view, it would be critical for a tumour to achieve a co-ordinated and finetuned TME establishment. In this case, a variation in the initial cancer cell-TME set up could result in an enduring mismatch that will ultimately impair tumour progression. However, 
74 dominant limiting factor for long term tumour growth, has never been formally tested. This is 75 due to the fact that the creation of the experimental set up to address this question is 76 extremely challenging. As a thought experiment, this would require interfering exclusively 77 with the extrinsic feature of a certain cancer cell without negatively influencing its intrinsic 78 tumorigenic potential. This change would induce an initial interaction with the in vivo 79 environment different from what would be typical for these cancer cells, which would in turn 80 generate an alternative TME. However, such perturbation induced in the cancer cells needs 81 to be reversible and the initial cancer cell characteristics fully recovered, in order to 82 effectively create the situation of a particular cancer cell type embedded in an alternative 83 microenvironment in vivo. In this scenario, the functional consequence of a mismatch 84 between cancer cells and their typical TME could be tested.

85 Here, we present an experimental setting where the secretion profile of the 86 aggressive murine breast cancer $4 \mathrm{~T} 1$ cells is perturbed by transitory inhibition of ROCK87 Myosin II signalling either via the pre-treatment with an inhibitor or the use of inducible shRNA. Crucially, ROCK-Myosin II inhibition, does not influence 4T1 growth ability in vitro.

89 Transiently less contractile 4T1 cells initiate an alternative TME compared to the one they 90 would normally create when grafted in the fat pad. Importantly, even if cancer cells recover 91 their ROCK-Myosin II activity early during tumour growth, we found that such alternative 92 TME is able to limit their long-term cancer growth due to the recruitment of an alternative 93 stroma and the generation of alternatively polarized anti-cancer macrophages. Therefore, 94 this work represents a direct experimental evidence that the creation of an early optimal 95 microenvironment is a dominant factor in the cascade of events determining long-lasting cancer growth and highlights the prognostic value of early detection of TME changes in the 97 clinic. 
Rock inhibition of murine 4T1 breast cancer cells alters their secretome without affecting their intrinsic growth potential

Human breast cancer lines show a variable level of intrinsic susceptibility to the highly specific ROCK inhibitor GSK269962 (from now on named ROCKi), and a number of cell lines do not show an intrinsic response to the drug when treated in vitro for 3 days (Figure 1A). Therefore, we firstly used ROCKi to select a murine cell line able to maintain intrinsic growth capacity upon ROCK inhibition. Cells were acutely exposed to ROCKi for 5 days and their growth in different conditions was assessed after treatment interruption (pROCKi) (Figure 1B). The lower levels of MLC2 phosphorylation (pMLC2) confirmed that ROCK inhibition was maintained for at least three days after stopping the treatment in the murine

112 4T1 breast cancer cell line (Figure $1 \mathrm{C}$ and D). While ROCKi in the 4T07 breast cancer line caused reduced growth long term (Supplementary Figure $1 \mathrm{~A}$ and $\mathrm{B}$ ), we found that $4 \mathrm{~T} 1$ cell growth in different in vitro conditions was not affected by pre-treatment with ROCKi (Figure $1 \mathrm{E}-\mathrm{G}$ and Supplementary Figure $1 \mathrm{C}$ and $\mathrm{D}$ ). Importantly, during this period, less contractile $4 \mathrm{~T} 1$ cells showed an alteration in various secreted factors found in the culture media (Figure $1 \mathrm{H}$ and I). We next wanted to assess if a perturbation in the secretome reflects the ability of cancer cells to engage into host interactions. As tumour associated fibroblasts are the most abundant and a key cellular component of the stroma in breast cancer(Friedman et al. 2020), we used classical fibroblast activation assays in vitro to functionally test the supernatant of ROCKi pre-treated cells. To exclude potential direct effect from the ROCKi, we confirm the absence of ROCKi in culture media of pre-treated cells by mass spectrometry (Supplementary Figure 1E). Interestingly, while both control and pROCKi-4T1 cancer cells similarly influence fibroblast proliferation in vitro, these fibroblasts displayed different functional characteristics such as morphology, extracellular matrix contraction and migration abilities (Supplementary Figure 1F-J). These data indicate that, in principle pROCKi-4T1 cancer cells altered normal fibroblasts' activation in vitro.

Collectively, these data suggests that the ability of ROCK signalling to modulate intrinsic and extrinsic functions could be effectively uncoupled in $4 \mathrm{~T} 1$ cancer cells, creating the ideal setting to test our hypothesis.

Transient inhibition of cancer cell contractility causes long-term growth inhibition in vivo

134 Next, we tested the capacity of $4 \mathrm{~T} 1$ cells pre-treated with ROCKi (pROCKi-4T1) to form 135 tumours when implanted in the fat pad of syngeneic Balb/c mice (Figure 2A). We found that 136 while the initial phase of tumour establishment (8 days post-transplantation) was unaffected, 
137 the long-term growth ability of pROCKi-4T1 cells was strongly compromised (from 14 days 138 post-transplantation) (Figure 2B-C). Importantly, at an early stage of tumour establishment 139 a time point where tumour growth was unaltered - the overall levels of pMLC2 were nearly 140 fully recovered from the ROCKi pre-treatment (Figure 2E and Supplementary Figure 2A-C). 141 This early bulk of growth is likely driven by the intrinsic ability of cancer cell growth, and it is 142 in line with the evidence that pROCKi-4T1 growth in vitro was not affected by a reduction in 143 MLC2 phosphorylation (Figure 1E-G and Supplementary Figure1C and D). Importantly, the long-term reduction of tumour growth resulting from transient inhibition in ROCK signalling was confirmed using an inducible shRNA against its direct ROCK target MYL9/MYL12B (genes encoding Myosin Light Chain 2) (Figure 2F-H). Decreased MLC2 expression was confirmed in cells before transplantation in the mice (Figure $2 \mathrm{G}$ ). The mice were maintained under doxycycline induction only for 5 days post-transplantation (Figure 2F). Similarly, to the results using ROCKi pre-treatment, early block in MLC2 expression resulted in an initial unperturbed growth (day 10), follow by a growth reduction (from day 14) and a long-term growth suppression (Figure $2 \mathrm{H}$ ).

High contractility is a feature of aggressive tumours where TME's characteristics were shown to actively sustain ROCK-Myosin II signalling and invasion of cancer cells (Northcott et al. 2018; Laklai et al. 2016) (Wong et al. 2020). As expected, the reduced level of growth in pROCKi-4T1 tumours at day 14 triggered a feedback loop where cancer cells show a lower level of MLC2 phosphorylation (Supplementary Figure 2D). This suggests the generation of an alternative TME that is less fit to support long term ROCK-Myosin II signalling, a feature of $4 \mathrm{~T} 1$ cells and, in turn, their typically aggressive growth. To test this hypothesis, we isolated cells from early-stage tumours, when tumour growth is not affected, and cancer cells have recovered MLC2 phosphorylation (Figure 2E). By magnetic cell sorting (MACS), we isolated either human CD2 (hCD2)-labelled cancer cells (A) or, alternatively, the rest of the TME depleted cells from the majority of cancer cells (B) (Figure 2l). By re-transplanting the isolated cells in new recipient mice, we tested the ability of 4T1 cells from pROCKi-4T1 tumours to re-establish secondary tumour growth as opposed to their ability to re-establish secondary tumours when maintained in the overwhelming presence of the TME they have previously established (Figure 2I). In line with the fact that cancer cells have recovered their original features (Figure 2E and Supplementary Figure 2AC), 4T1 cells from pROCKi-4T1 tumours show no delay in sustaining long term growth upon re-transplantation compared to control cells (Figure 2J). Strikingly, when the same cancer cells were re-transplanted together with the previously established TME, 4T1 cells from pROCKi-4T1 tumours maintained a strong reduction of tumour growth in secondary recipient already from early stage (Figure $2 \mathrm{~K}$ ). These results point at a dominant inhibitory effect of 
173 the TME generated in pROCKi-4T1 tumours on 4T1 cells, which otherwise possess an 174 unaltered growth capacity.

177 We next analysed the TME cellular composition of tumours from control and pROCKi-4T1

178 cells after cancer establishment. Early pROCKi-4T1 tumours showed an increase in $\alpha \mathrm{SMA+}$ 179 stromal cells, one of the dominant TME component in control 4T1 tumours (Figure 3A and 180 B). In order to analyse the characteristics of the TME we isolated the general stromal compartment, depleted from immune-cells and endothelial cells, from control and pROCKi4T1 tumours (Figure $3 \mathrm{C}$ ) at the early time point, when no growth delay was detected. Deep RNA sequencing showed an alternative signature of stromal cells from pROCKi-4T1 compared to stromal cells isolated from control tumours (Figure 3D and E). However, when we functionally tested these alternatively activated stromal cells for their ability to support cancer cell growth, no difference was detected neither in 3D co-culture system nor in coinjection assay in vivo (Supplementary Figure 3A-D). Similar results were observed when co-injecting in vitro activated fibroblasts previously shown to display perturbed characteristics (Supplementary Figure $3 \mathrm{E}$ and $\mathrm{F}$ and Supplementary Figure 1F-J). This suggests that these alternative activated stromal cells do not show anti-tumour effect per se, but they reflect the early generation of an alternative TME initiated by pROCKi-4T1 cells. When analysing the features of the alternative TME captured by its stromal signature, we found that extracellular matrix (ECM) regulation and integrin signalling were upregulated 194 (Figure 3E) pointing at changes within the non-cellular ECM compartments of the TME. We could indeed detect an increase in matrix density and Collagen IV staining in the pROCKi4T1 tumours compared to control (Supplementary Figure $3 \mathrm{G}$ and $\mathrm{H}$ ). Importantly, the stromal signature found in tumours generated by pROCKi-4T1 cells with reduced contractility, named Cancer Low Contractility (CLC)-TME signature, showed strong prognostic power to identify human breast cancer patients with better survival in TCGA database (Figure 3F-H). This indicates that this alternative activated stromal signature characterized less aggressive human breast cancers. Indeed, this signature shows downregulation of pathways key for CAF function (Figure $3 \mathrm{E}$ ), suggestive of a failure to establish the suitable pro-tumoral activity needed in the TME of aggressive and fast-growing tumours.

Alternatively activated stroma from ROCKi pre-treated tumours shows different immuno-interaction activities

208 Bioinformatics analysis showed that a major difference in the enriched pathways in pROCKi209 4T1 tumour's stroma was inflammatory signalling pathways/chemokines and cytokine 
210 release, suggesting broad alterations within the inflammatory compartment of the TME 211 (Figure 3E). Consistently, when the immune-cell composition of tumours was analysed by 212 FACS, we found significant differences in control or pROCKi-4T1 tumours specifically at 213 later time points (Figure $4 \mathrm{~A}-\mathrm{H}$ ). Data showed a decrease in cells from the lymphoid lineage 214 in favour of an increase in myeloid cells.

215 To test if a decrease in tumour infiltrating lymphocyte could contribute to the delay in tumour 216 growth, control or pROCKi-4T1 cells were transplanted in immune-compromised RAG1-/217 mice, lacking B and T lymphocytes (Figure 4I). Similar delays in the growth of pROCKi-4T1 218 tumours were observed, excluding an involvement of the adaptive immune system to limit 219 sustained tumour growth (Figure 4J).

220 Macrophages are known to possess both pro- and anti-tumorigenic functions (Noy \& Pollard 221 2014), therefore we tested if a different polarization in the macrophage compartment could 222 be detected in pROCKi-4T1 tumours. Tumour associated macrophages (TAM) showed no difference in canonical markers that have been previously associated to pro- or anti-cancer activities (Supplementary Figure 4A and B). Accordingly, when analysing the immuneinfiltrates in the TCGA human breast cancers classified according to the pROCKi-4T1 tumours CLC-TME signature (Figure 3F), there was no difference in M1 or M2 macrophages (Supplementary Figure 4C). These data show that the change in the inflammatory status of the pROCKi-4T1 TME does not translate into a classical macrophage pro-tumour polarization.

pROCKi-4T1 tumours showed the presence of macrophages with non-canonical anticancer activation in vitro

233 We next aimed to determine if, despite showing canonical TAM markers, macrophages in pROCKi-4T1 tumours, harbour different characteristics. To do this, we generated a gene expression signature of $\mathrm{F} 4 / 80$ macrophages isolated from control or pROCKi-4T1 tumours (Figure 5A). Striking differences were detected at the gene expression level (Figure 5B and C). Interestingly, the emergence of a distinct signature was detected 8 days posttransplantation and was consolidated at later time points, when tumour growth was significantly reduced (Figure $5 \mathrm{~B}$ and $\mathrm{C}$ ).

240 Analysis of the macrophage signature from the two types of tumours using GSEA revealed 241 an increase in anti-tumour inflammation signalling Interferon gamma (INF $\gamma$ ) and a decrease 242 in pro-tumour inflammation signalling NFKB signalling in pROCKi-4T1 tumour derived macrophages (Figure 5D), pointing to an anti-tumour polarization. Importantly, when we analysed the conditioned media of stromal cells isolated from pROCKi-4T1 tumours at 14 days, we detected a dramatic decrease in specific secreted factors cytokines compared to 
control tumours (Supplementary Figure 5A). Particularly, at this tumour stage, network enrichment analysis supported the overall decrease in the NFKB signalling also in stromal cells (Supplementary Figure 5B), which was previously reported to be critical for CAFmediated enhancement of tumour promoting inflammation (Erez et al. 2010). These differences observed in stromal cells cytokine secretion sampled a global alternative microenvironment promoting alternative inflammatory pathways. The activation of INF $\gamma$ activity indicated by the signature of infiltrating macrophages of pROCKi-4T1 tumours, was confirmed by a sharp increase in active STAT1 nuclear staining at late time points (Figure 5E and F). Importantly, similar PSTAT1+ macrophages were detected at the late stage of growth in tumours initiated by cells initially depleted in MYL9/12b (Figure 5G and Figure $2 \mathrm{~F}-\mathrm{H}$ ). Interestingly, already at early stages, infiltrating macrophages of pROCKi-4T1 tumours sowed increased pSTAT1 staining (Supplementary Figure 5C). Moreover, as predicted by their signature, we also measured a reduction in nuclear p65 (RelA component of the NFKB complex) in macrophages from pROCKi-4T1 tumours (Supplementary Figure 5D), which results in an overall switch from NFKB to INF $\gamma$ signalling activity (Supplementary Figure 5E). We therefore hypothesise that macrophages undergo profound transcriptional re-programming that inhibits their pro-tumorigenic potential in the pROCKi$4 \mathrm{~T} 1$ tumours.

264 To assess the functional implications of such alternative activation in macrophages, we tested their direct interaction with cancer cells by using 3D co-culture scaffold system to better model the cell-cell interactions ex vivo. As we used freshly isolated macrophages in the assay, we also used primary breast tumour cells isolated from spontaneous ActinGFP/MMTV-PyMT breast tumours in the co-culture. Freshly isolated macrophages from either control or pROCKi-4T1 tumours show no differential activity when co-cultured alone with cancer cells seeded on 3D scaffolds (Supplementary Figure 5F-H). However, when cancer cells were seeded together with their cancer associated stromal cells, macrophages isolated from 14 days pROCKi-4T1 tumours showed a cancer growth inhibitory effect (Figure

$2735 \mathrm{H}$ and $\mathrm{I}$ ). Similar results were observed in 2D culture conditions (Figure 5J, 5K). 274 Interestingly, fibroblasts supported the cancer cell killing activity of alternative polarized macrophages from pROCKi-4T1 tumours, yet they do not show the power to influence this activity ex vivo. Indeed, co-culture of fibroblasts from pROCKi-4T1 tumours with control (DMSO) macrophages did not induce killing activity (Figure 5K). These data suggest that the alternative anti-tumour polarization observed in macrophages from pROCKi-4T1 is

279 progressively established within the complex in vivo TME and it becomes a hardwired 280 status. 
282 Non-canonical macrophages are responsible for limiting pROCKi-4T1 tumour growth 283 in vivo

284 To test whether this alternative anti-tumour polarization of macrophages was acting to limit 285 cancer cell growth in vivo, we used clodronate to deplete macrophages from control or 286 pROCKi-4T1 cell transplants (Figure 6A). In line with the known pro-tumour activity of 287 tumour infiltrating macrophages in breast cancer (Noy \& Pollard 2014), their depletion 288 decreased the final size reached by control tumours (Figure 6B). However, an opposite 289 effect was shown in pROCKi-4T1, where their depletion boosted overall tumour growth and 290 rescued it to the level of control tumours (Figure 6C). This data highlighted that the 291 alternative macrophage polarization we described, indeed results in a switch from pro- to 292 anti-tumour function.

293 We have shown in Figure 3 that the activated stroma from pROCKi-4T1 cells harbour a gene 294 signature (CLC-TME signature) with prognostic value in human breast cancer. Similarly, we 295 generated the macrophages CLC-TME signature using the downregulated genes in 296 macrophages from pROCKi-4T1 tumours, initiated from cancer cells with reduced 297 contractility. Strikingly, we found that the macrophages CLC-TME signature showed a 298 similar power in predicting better survival of human breast cancer patients (Figure 6D). 299 Moreover, 43\% of these human tumours additionally showed the alternative stromal CLC300 TME signature, suggesting a similar correlation between stromal cells and macrophages 301 characteristics in the tumour microenvironment can be found in human breast cancers with 302 better prognosis (Figure 6E). Importantly, as no difference was found in macrophage 303 markers generally associated to anti-tumour phenotypes in breast tumours (Supplementary 304 Figure 4C), this data rises the possibility of the possible presence of a previously 305 unappreciated alternative type of anti-tumour macrophages also in human cancers, which 306 correlates with better clinical outcome. 


\section{Discussion}

In this work, we provide evidence that ROCK-Myosin II activation in cancer cells with basal characteristics represents an important modulator for the initiation of their characteristic TME in vivo. When cancer cells have high ROCK-Myosin II activation, this is generally required for their intrinsic tumourigenicity (Rodriguez-Hernandez et al. 2020; Kümper et al. 2016), but we identify a murine cell line which represented an exception. Indeed, the aggressive murine 4T1 breast cancer maintained its highly proliferative activity in various in vitro conditions upon ROCK-Myosin II inhibition but showed a perturbed fibroblast activation ability. After identifying the 4T1 as a cell line where the intrinsic and extrinsic ROCK-Myosin II activity could be uncoupled, we used a transient ROCK-signalling inhibitor (GSK269962A) (ROCKi) treatment and inducible shRNA strategy to showed that initially less contractile 4T1 cells are less capable of sustained long-term growth in vivo. Importantly, this delay was maintained even after recovering ROCK-signalling activation. Indeed, secondary transplantation experiments show that, while cancer cells recover their growth capacity, the TME generated by initially less contractile $4 \mathrm{~T} 1$ cells is dominant and inhibits their growth. Our data support the fact that ROCKi pre-treated cancer cells initiate an alternative TME characterized by an increase early representation of SMA positive cells. Moreover, the stromal component from initially less contractile tumours showed an alternative signature. Indeed, secretome analysis on ROCKi pre-treated 4T1 conditioned media revealed significant differences with control 4T1 media in the secretion of 20 cytokines (where LIF, WISP1, MMP9 and several others resulted downregulated in pROCKi), some of which have been previously linked to CAF activation. For instance, cancer derived LIF was shown to promote CAF activation and pro-invasive phenotype via inducing actomyosin contractility and ECM remodelling (Albrengues et al. 2014). In addition, WISP1 was found in the metastatic niche and was required for breast cancer metastasis to the lung (Ombrato et al. 2019). Thus, it is tempting to speculate that such a differential cocktail of factors could interfere with the pro-tumorigenic stromal activation generally induced by $4 \mathrm{~T} 1$ cells. Despite the transcriptomic analysis of stromal TME component from control or ROCKi pretreated tumours shows an alternative gene-expression signature, they similarly supported cancer cell growth ex-vivo and in vivo, excluding a direct anti-tumour activity. However, this alternative stromal component is part of a global TME change, which ultimately results to be unfit to support aggressive tumour growth. Indeed, when analysing the secretome of stromal cells from ROCKi pre-treated cancer the downregulation of NFKB signalling emerged, which was previously reported to be critical for CAF-mediated enhancement of tumour promoting inflammation (Erez et al. 2010). When analysing the tumour infiltrates, we identified a reduction on lymphocytic cells (B and T-cells, specifically CD4+ but not CD8+ cells) and an 
increase in myeloid cells (macrophages and dendritic cells). We excluded the role of the lymphocytic compartment in the observed phenotype as ROCKi pre-treated cancer cells

347 maintained their deficit in growth in genetically-immunodeficient mice lacking mature 348 lymphocytes (Rag1-/-).

349 Although tumour associated macrophages (TAMs) did not present distinct expression of canonical pro- or anti- tumour markers between tumours from control and ROCKi pre-treated cancer cells, RNAseq analysis showed very broad changes in their signature. In particular, TAMs from tumours generated by ROCKi pre-treated cancer cells suggested an anti-tumour function with a significant increase in Interferon $\gamma(\mathrm{INF} \gamma)$. Indeed, TAM infiltrating ROCKi pretreated tumours showed clear increase in nuclear PSTAT1, the effector transcription factor downstream of INF $\gamma$.

We could confirm that this anti-tumorigenic function was responsible for inhibiting cancer growth in ex-vivo and in vivo experiments. Interestingly, the TAM anti-tumour function in exvivo co-culture was only detected in presence of freshly isolated stromal cells. Interestingly, alternatively activated stroma isolated from ROCKi pre-treated cancer cells' tumours had no effect on the ex vivo behaviour of TAMs, suggesting that macrophage polarization acquired intra-tumorally is a stable phenotype. Importantly, when testing the functional role of macrophages on tumour growth in vivo we confirmed their anti-tumour function. By using Clodronate, a type of bisphosphonate which once encapsulated into liposomes is up taken by macrophages and mediates their depletion (Van Rooijen \& Hendrikx 2010), we could clearly see the opposing effects in control or ROCKi pre-treated tumours in either inhibiting or promoting growth respectively.

Thus far, it has been shown how CAFs can contribute to assembling an immunosuppressive TME accordingly to their type of activation (Costa et al. 2018). We could detect an alteration in pathways responsible with immunomodulation in CAFs. We found macrophages infiltrating ROCKi pre-treated tumours to display increased INF $\gamma$-STAT1activation and reduced NFkB signalling. This was shown by the increase in nuclear staining for the INF $\gamma$ effector pSTAT1 and reduced staining for p65. INF $\gamma$ signalling is typical of anti-tumour functions in the immune microenvironment (Katlinski et al. 2017), therefore a switch in signalling activation could control macrophage behaviour.

Collectively, the present study reveals a key role for the actomyosin cytoskeleton of cancer cells in setting out an early functional TME (Figure 7). When actomyosin contractility is inhibited in cancer cells during early phases of in vivo growth, a different TME emerges that is characterised by an alternative early stromal activation, which represent one of the more abundant TME components. We detected a broad lack of pro-tumoral signals and a 
381 signatures (Figure 7). Remarkably, the anti-tumour TAMs CLC-TME signature, similarly to 382 the fibroblasts CLC-TME signature, allows identification of human breast cancers with better 383 prognosis. Similarly, to TAMs found in tumours from ROCKi pre-treated cells which do not 384 show canonical M1 markers, no M1 macrophage infiltration was observed in human tumours 385 correlating with the alternative fibroblast and macrophage signature, suggesting that this 386 non-canonical anti-tumour macrophage polarisation might exist in human tumours and 387 contribute to better clinical outcome.

388 Overall, our data shows that cancer cells assemble specific tumour 389 microenvironments very early in tumour development. Optimal assembly is crucial for 390 tumour growth and perturbations of the cytoskeleton of cancer cells diminish their ability to 391 establish the right stromal and immune partners for successful tumour progression. This 392 early deficit becomes dominant, as the alternative TME results unsuitable for long term 393 growth, firstly due to the inability of the cancer cells to foster aggressive features, for 394 instance by failing to fully recover their contractility at the later stages of growth. Ultimately, 395 this cascade of event leads to the generation of anti-tumour macrophages which effectively 396 halt tumour growth. In conclusion, our data show that definition of the early 397 microenvironment is key for long term grow, likely due to the constant crosstalk occurring 398 between cancer cells and their local ecosystem. Therefore, early detection of TME changes 399 could harbours a strong prognostic value in the clinic. 
401 Figures and Legends

402 Figure 1

403

\section{Figure 1}

405 A. Distribution of ROCKi response across human breast cancer cell lines. IC50 of 406 GSK269962A inhibitor across 48 human breast cancer cell lines. Compounds are ranked in 407 descending order of response and divided into responders (IC50<5 $\mu \mathrm{M}$, red), intermediate

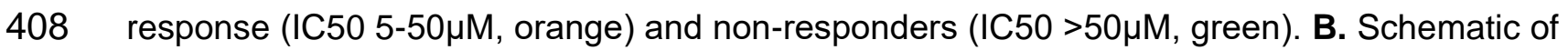
409 the experimental setup for the characterisation of the intrinsic effects of GSK269962A on 410 cancer cells. Cancer cells were plated and treated daily with vehicle (DMSO) or $5 \mu \mathrm{M}$ of 411 GSK269962A ROCK1/2 inhibitor (ROCK1) for five days. C. Western blot analysis of 412 Phospho-MLC (pMLC) after 5 days of ROCKi treatment (5 Days), 24h or 72h post-treatment. 413 (Left panel) Quantifications of relative pMLC2 levels in 4T1 cells as a fold change versus the 414 control from two independent experiments (right panel) D. Immunofluorescence images of 415 pre-treated $4 \mathrm{~T} 1$ cells 72 hours after treatment was removed. Cytoskeleton was stained using 416 phalloidin (red), contractility using pMLC (green) and nucleai using DAPI (blue). (Scale bar $41750 \mu \mathrm{m})$. E. Representative brightfield images of $4 \mathrm{~T} 1$ cells following a 5 -day treatment with 418 GSK269962A inhibitor. (Scale bar $100 \mu \mathrm{m}$ ). F. Growth curve of cells pre-treated with DMSO 419 (black) or GSK269962A inhibitor (red). Cell confluency was monitored every 3 hours for $420<120$ hours using Incucyte. G. Percentage of cell viability quantified after 5 days of treatment 421 and at day 4 post-treatment: DMSO (black) or GSK269962 (red) for 5 days. Error bars 422 represent the SEM from three independent experiments. Statistic, Student's t-test ${ }^{*} p<0.05$, $\left.423{ }^{* *} p<0.01\right)$. H. Quantification of differentially secreted factors in the conditioned media using 424 Protein Array () of pre-treated 4T1 cells, 48 hours after treatment. Error bars represent SEM 425 from two technical replicates. I. Heatmap of the top 3 upregulated (red) and downregulated 426 (green) factors in GSK269962A-conditioned media. 


\section{Figure 2}

429

\section{$430 \quad$ Figure 2}

431 A. Schematic of the experimental setup. B. Mean tumour volume over time of tumours 432 generated by 4T1 cells pre-treated with DMSO (black) or GSK269962A (red) in the fat pad 433 of Balb/cAnN mice. Error bars represent SEM from four independent experiments (total of $434 \mathrm{n}=20$ tumours). Statistic two-way ANOVA with Sidak's multiple comparisons test C-D. 435 Histogram of tumour weight at day $8(n=28)(C)$ and day $14(n=12)(D)$. Statistic: Student's t436 test between DMSO and GSK269962A groups. Statistically significant (ns: not significant, $437{ }^{* * *} p<0.0001 \mathrm{E}$. (Left) H-score of $\mathrm{p}-\mathrm{MLC} 2$ staining in 4T1 tumour-derived cells pre-treated 438 with DMSO (blue) or GSK269962A (red) from days 5 and 8 (Right) Representative QuPath 439 color-maps of pMLC2 staining in both conditions. F. Schematic of the experimental setup: 440 Doxycyline was administered to the cells in vitro and in vivo during the first five days post441 transplantation into the fat pad of Balb/cAnN mice. G. Western blot analysis of MLC2 after 5 442 days of DOX treatment in vitro. $\mathbf{H}$. Mean tumour volume of $4 \mathrm{~T} 1$ cells transduced with a 443 hairpin inducible vector for Non-targeting (left, black/grey) or MYL9/12b-targeting shRNA 444 (right, purple/pink). Error bars represent SEM from two independent experiments ( $\mathrm{n}=8$ total 445 number of tumours). Data were analysed using two-way ANOVA with Sidak's multiple 446 comparisons test. (ns: not significant, ${ }^{*} p<0.05$ ). I. Schematic of the experimental setup. 447 Day 8 tumours were separated by magnetic cell sorting into (A) cancer cells (hCD2+) and 448 (B) tumour microenvironment (TME, hCD2-). Each of the components, was separately 449 injected into new naïve Balb/cAnN mice. J. Mean tumour volume of (A) 4T1 cells isolated 450 from day 8 DMSO (black) or GSK269962A (red) tumours. Error bars represent SEM from 451 two independent experiments ( $n=9$ total number of tumours). K. Mean tumour volume of (B) 452 TME cells isolated from day 8 DMSO (black) or GSK269962A (red) tumours. Error bars 453 represent SEM from two independent experiments ( $n=7$ total number of tumours). Data were 454 analysed using two-way ANOVA. (ns: not significant, ${ }^{* * *} p<0.001$ ). 


\section{Figure 3}

457

\section{Figure 3}

459 A. Representative IHC images of orthotopic syngeneic tumours 8 days post-transplantation 460 from 4T1 pre-treated with DMSO or ROCKi. Hematoxilin/Eosin (left), $\alpha$ SMA antibody DAB461 staining (middle) and fluorescence double staining of aSMA (green), Endomucin (red) and 462 DAPI (blue) to stain the nuclel (right) (Scale bar $100 \mu \mathrm{m}$ ). B. Quantification of $463 \alpha \mathrm{SMA}+$ Endomucin- cells in DMSO or ROCKi pre-treated tumours 8 days post464 transplantation. Error bars represent the SEM from 4 tumours with 6 fields of view 465 (FOV)/tumour. Data were analysed using a student's t-test $\left({ }^{*} p<0.05\right)$. C. Experimental 466 design for tumour associated mesenchymal cell isolation. D. Volcano plot comparing the 467 gene expression profile of stromal cells derived from ROCKi vs. DMSO pre-treated tumours. 468 Statistical significance (-log10 adjusted pvalue) against foldchange (log2) of fibroblasts 469 derived from ROCKi vs. DMSO pre-treated tumours. Significantly upregulated genes are 470 shown in red and downregulated genes are shown in blue. E. Gene signatures in CAF471 specific molecular mechanisms were selected and ranked (upregulated in red; 472 downregulated in blue). Gene-expression differences are displayed as a normalised 473 enrichment score (NES), and false discovery rate (FDR) is used to determine the 474 significance of each pathway. The size of the circle represents the number of genes within 475 each signature that are differentially expressed in ROCKi vs. DMSO-CAFs. F. (Left panel) 476 Schematic representation of how the Cancer Low Contractility (CLC) - TME signature was 477 generated stemming from genes downregulated in tumour initiated by cancel cells with 478 reduced contractility (ROCKi). Padjusted value (Padj) and log2 fold change (fold change) 479 were used as cut-offs for gene selection. (Right panel) Heatmap of the 46 genes that 480 constitute the CLC-TME signature and their expression across 1092 breast cancer tumours 481 from the TCGA cohort. G. Log2 expression of the CLC-TME signature divided patients into 482 high (red, equivalent to signature in mouse control tumours) and low (blue, equivalent to 483 signature in mouse ROCKi tumours) quartiles. Box-an-whisker plots showing median centre 484 line, $25 \%$ and $75 \%$ box limits and rang of expression. $\mathbf{H}$. Kaplan-Meier curve represents the 485 overall survival of 546 breast cancer patients from the TCGA cohort subdivided into high 486 (red) and low (blue) expression of the ROCKi-CAF "down signature". Data were analysed 487 using a Wald test and statistically significant changes between the two groups are indicated $488 \quad\left({ }^{*} p<0.05\right)$ (hazard ratio is 1.96 and confidence intervals are $\left.1.1-3.93\right)$. 


\section{$490 \quad$ Figure 4}

$491 \quad$ Figure 4

492 A. Experimental design and antibodies used for detecting immune cells populations within 493 the TME. B-H. Flow cytometric quantification of TME cell frequencies out of the total alive 494 cells lungs in mice harbouring DMSO (grey) or GSK269962A (ROCKi) (red) pre-treated 495 tumours at the indicated times post-injection. (B) total CD45+ immune cells (C) CD45+CD3+ 496 T-cells; (D) CD45+CD4+ T-cells; (E) CD45+CD8+ T-cells; (F) CD45+CD19+ B-cells; (G) 497 CD45+CD11c+ dendritic cells; $(H)$ CD11b+F4/80+ macrophages. Error bars represent the 498 SEM from two independent experiments (Total $n=12$ in each group). Data were analysed 499 using a t-test between DMSO and ROCKi groups at each time points. $\left({ }^{*} p<0.05\right.$, ${ }^{* *} p<$ 500 0.001). I. Diagram of the experimental design. J. Mean tumour volume of 4T1 cells grown 501 over 26 days. Tumours were pre-treated in vitro with DMSO (black) or ROCKi (red). Error 502 bars represent the SEM from two independent experiments (total of $n=12$ (DMSO) and $n=10$ $503(\mathrm{ROCKi}))$. Data were analysed using a two-way ANOVA ( $\left.{ }^{* * *} p<0.0001\right)$. 


\section{Figure 5}

506

\section{Figure 5}

508 A. Schematic representation of the experimental design and sample collection. B. Heatmap 509 comparing the gene expression profile of macrophages derived from ROCKi vs. DMSO pre510 treated tumours 8 and 14 days post-transplantation. C. Volcano plots of statistical 511 significance (-log10 adjusted pvalue) against foldchange (log2) of macrophages derived 512 from ROCKi vs. DMSO day 8 pre-treated tumours (Left) or day 14 pre-treated tumours 513 (Right). Significantly upregulated genes are shown in red and downregulated genes are 514 shown in blue. D. Summary of selected pathways enriched in ROCKi vs. DMSO. 515 Upregulated or downregulated pathways at the indicated time point are indicated in in red 516 and blue respectively.

517 E. Percentage of pSTAT1 positive macrophages derived from DMSO (grey) or ROCKi (red) 518 pre-treated tumours from day 14. F. Representative staining of F4/80 (yellow) and pSTAT1 519 (Magenta) of DMSO or ROCKi pre-treated tumours section (Scale bar $50 \mu \mathrm{m}$ ). G. Percentage 520 of PSTAT1 positive macrophages derived from control or DOX treated of non-targeting or 521 MYL9/12b-targeting shRNA (Fig. 2F-H). H. Experimental design of the co-culture. I. 522 Quantification of primary MMTV-PyMT-GFP+ cell growth by measuring the total GFP+ area 523 over the course of 4 days. Cells were grown with DMSO-Macrophages and CAFs (grey) or 524 ROCKi-macrophages and CAFs (red) from 14-days tumours and normalised to the growth of 525 MMTV-PyMT cells alone (dash line). Representative fluorescence pictures are shown. Error 526 bars represent the SEM from three independent experiments shown with black, green or red 527 data points. Biological replicates within each experiment are indicated in the graph. Data 528 were analysed using a Student's t-test $\left({ }^{*} p<0.05\right)$. J. Representative brightfield images of 529 MMTV-PyMT-GFP+ cells (green) in co-culture with ROCKi-CAFs and either DMSO (left) or 530 ROCKi (right) macrophages. Timepoints are indicated. Scale bar, $30 \mu \mathrm{m}$. K. Quantification of 531 MMTV-PyMT-GFP + cell area when cells were grown alone (black), with macrophages from 532 DMSO (grey) or ROCKi (coral) pre-treated tumours or with CAFs from ROCKi pre-treated 533 tumours and macrophages extracted from either DMSO (blue) or ROCKi (red) pre-treated 534 tumours. Statistic two-way ANOVA (blue vs red) $\left({ }^{* * *} p<0.0001\right)$. 


\section{Figure 6}

\section{Figure 6}

538 A. Experimental design. B-C. Mean volume of $4 \mathrm{~T} 1$ tumours over 22 days. $4 \mathrm{~T} 1$ tumour cells

539 were pre-treated in vitro with DMSO (B) or ROCK inhibitor $(\mathrm{C})$, and tumours were treated

540 with either PBS liposomes (black and red lines) or clodronate liposomes (purple lines). Error

541 bars represent the SEM ( $\mathrm{n}=8$ tumours per condition). Data were analysed using a two-way

542 ANOVA comparing the curves (at the end of the curves) (ns: not significant, ${ }^{*} p<0.05$ ) and

543 t-tests at individual timepoints when significant difference were present (indicated above

544 each time point) ( $p<0.05$ ). D. Kaplan-Meier curve represents the overall survival of 546

545 breast cancer patients from the TCGA cohort subdivided into high (red) and low (blue)

546 expression of the macrophage signature. Data were analysed using a Wald test and

547 statistically significant changes between the two groups are indicated $\left({ }^{*} p<0.05\right.$ ) (hazard

548 ratio is 1.96 and confidence intervals are $1.1-3.93)$. E. Pie charts represent the overlap

549 between patients with both alternative macrophage and alternative stromal signatures from

550 ROCKi pre-ptreated tumours.

551

\section{$552 \quad$ Figure 7}

\section{Figure 7}

554 Graphic summary: breast cancer cells with initial reduced ROCK-Myosin II activity generated 555 an alternative early TME characterized by alternative stroma displaying a Cancer Low 556 Contractility (CLC)-stromal-signature. The CLC-stromal-signature show overall reduced pro557 inflammatory activity including down-regulation of $\mathrm{NF} \kappa \mathrm{B}$ signalling. The alternative TME fails 558 to sustain pro-tumourigenic signalling typical of Tumour Associated Macrophages (TAM), 559 which consequently display a TAM CLC-signature with reduced pro-tumoural NFKB 560 signalling and anti-tumour Interferon Gamma (INF $\gamma$ ), STAT1 ${ }^{\text {high }}$ activity. These macrophages 561 -lacking canonical M1 markers- reduced long-term tumour growth. 


\section{Supplementary Figures and Legends}

\section{Supplementary Figure 1}

566

\section{Supplementary Figure 1}

568 A. Representative brightfield images of $4 \mathrm{~T} 07$ cells following a 5-day treatment with ROCKi.

B. (Left) Growth curve of cells pre-treated with DMSO (black) or ROCKi (red). Cell confluency was monitored every 3 hours for $<120$ hours using Incucyte. (Reft) Percentage of cell viability quantified at 5 days after treatment and at day 4 post-treatment: DMSO (black)

572 or ROCKi (red) for 5 days. (Scale bar $100 \mu \mathrm{m}$ ). (B-D) Error bars represent the SEM from

573 three independent experiments (dots). Student's t-test analysis between DMSO and ROCKi

574 groups $\left({ }^{* * *} p<0.0001\right)$. C. Representative image of the surviving fraction of $4 \mathrm{~T} 1$ breast

575 cancer cell lines pre-treated with DMSO (black) or ROCKi (red) following weekly re-plating

576 and during three consecutive weeks. D. Representative bright-field images of pre-treated 577 4T1 cells with DMSO (upper panels) or ROCKi (lower panels) plated on plastic (Left), on a 578 Collagenl-matrigel matrix (Middle) or embedded in matrigel (Right). (Scale bars $50 \mu \mathrm{m}$ ). E. 579 Liquid chromatography-mass spectrometry chromatograms on positive mode (green) or 580 negative mode (blue) on media containing 5uM of ROCKi as positive control (Left) and the 581 4T1-ROCKi pre-treated conditioned-media (Right). The detection limit of $0.01 \mathrm{nM}$ was 582 experimentally determined by diluting the positive control. F. Growth curve of mNBFs 583 incubated with DMSO (black) or ROCKi (red) conditioned media. Cell confluency was 584 monitored every 3 hours for $<150$ hours using Incucyte. Data were analysed using two-way 585 ANOVA test (ns: not significant). G. Representative brightfield images of (mNBF) cell morphology following a 2-day incubation with conditioned media (CM) from DMSO or ROCKi pre-treated 4T1 cells. (Scale bar $100 \mu \mathrm{m}$ ). H. Flow cytometric analysis of the size of mNBFs from (B) based on forward/side scatter. I. (Left) Schematic representation of the set-up for fibroblast contraction assays, (middle) representative pictures and (right) quantification of fibroblast contraction assays with DMSO (black) or ROCKi (red) pre-treated 4T1 cells. Error bars represent the SEM from three independent experiments and technical repeats (Total $592 \mathrm{n}=10$ per condition). Student's t-test analysis ( $\left.{ }^{* * *} \mathrm{p}<0.0001\right)$. J. (Left) Representative 593 example and (right) quantification of a murine normal fibroblast mNBF chemotaxis migration 594 assay towards DMSO (black) or ROCKi conditioned media from 4T1 pre-treated cells. 595 Fibroblast migration data was acquired every hour for 24 hours using Incucyte and the values are given in arbitrary units (A.U.). Data were analysed using two-way ANOVA test $\left({ }^{* * *} p<0.001\right)$. 


\section{Supplementary Figure 2}

602 A. Schematic of sample collection workflow where hCD2+ cancer cells were isolated from 603 tumours before performing a western blot. B. Representative western blot of pMLC2 and 604 GAPDH as loading control in $4 \mathrm{~T} 1$ pre-treated cells isolated from day 8 tumours. C. 605 Quantification of relative ppMLC2 levels in 4T1 tumour-derived cells pre-treated with DMSO 606 (black) or ROCKi (red). Error bars represent the SEM from two independent experiments 607 (total $n=6-7$ tumours). Student's t-test analysis (ns=non-significant). D. (left) Quantification of 608 H-score for p-MLC2 staining in 4T1 tumour-derived cells pre-treated with DMSO (blue) or 609 ROCKi (red) from day 14. (right) Representative QuPath color-maps of pMLC2 staining in 610 both conditions. Error bars represent the SEM from three independent experiments (total $611 n=17$ tumours/condition). Student's t-test analysis $\left({ }^{* * *} p<0.0001\right)$. 


\section{Supplementary Figure 3}

614

\section{Supplementary Figure 3}

616 A. Experimental design of co-culture on Alvetex scaffolds. B. Quantification of MMTV-PyMT617 GFP + cell growth using the total GFP+ area as a readout. Cells were grown alone (black), 618 with DMSO-CAFs (light grey) or ROCKi-CAFs (dark grey). Error bars represent the SEM 619 from three independent experiments shown data points. Biological replicates within each 620 experiment are indicated with black, blue or red in the graph. Student's t-test analysis 621 (ns=non-significant). C-D. Diagrams of the experimental design indicating the tumour origin 622 of the CAFs before co-injection with cancer cells (C). Mean tumour volume of 4T1 cells co623 injected with DMSO-CAFs (black) or ROCKi-CAFs (red) and grown over 24 days (D). Error 624 bars represent the SEM $(n=6)$, Data were analysed using two-way ANOVA (ns=non625 significant). E-F. Experimental design to generate activated fibroblasts in vitro before co626 injection with cancer cells (E). Mean tumour volume of MMTV-PyMT cells alone (purple) or 627 co-injected with DMSO-fibroblasts (black) or ROCKi-fibroblasts (red) and grown over 45 628 days $(F)$. Error bars represent the SEM $(n=6)$. Data were analysed using two-way ANOVA 629 (ns=non-significant). G. Representative IHC images of 4T1 tumours 8 days post-injection 630 stained with Masson's thricrome staining, marking keratin and muscle fibers (red), collagen 631 (blue), and cell nuclei (dark purple). Skin was used as a staining control. H. Representative 632 IHC images of 4T1 tumours 8 days post-injection stained collagen IV staining (brown). 633 Normal mammary gland tissue was used as a control. 


\section{Supplementary Figure 4}

\section{Supplementary Figure 4}

637 A-B. Flow cytometric quantification of pro-tumorigenic (CD206, CD163 and Arg1) (A) and 638 anti-tumorigenic (CD86, MHCII and iNOS) (B) macrophage polarisation markers 8- and 14639 days post-transplantation. Cells were gated on alive $\mathrm{F} 4 / 80+$ and error bars represent the 640 SEM from two independent experiments ( $n$ numbers indicated under each graph). Data were analysed using t-test $\left({ }^{* *} p<0.01\right)$. C. Cibersort analysis of the relative percentage of

642 immune cell populations in the 25 patients with higher (right - top samples) and lower (left -

643 bottom samples) cumulative gene expression for the down fibroblast signature from 644 Figure 3G. Analysis was performed in the TCGA cohort, and the figure legend (right) 645 indicates the cell populations investigated.

646

\section{Supplementary Figure 5}

\section{Supplementary Figure 5}

649 A. Heatmap displaying top differentially secreted factors in the fibroblasts from DMSO and 650 ROCKi-pre-treated (GSK) tumours at 14 days using comparative marker selection in 651 GennePattern. B. Network analysis enriched in ROCKi-pre-treated fibroblasts using 652 Ingenuity pathway analysis (RNA signature from Figure 3D). C. Percentage of pSTAT1 653 positive macrophages derived from DMSO (grey) or ROCKi (red) pretreated tumours from 654 day 8. D. Percentage of p65 positive macrophages derived from DMSO (grey) or ROCKi 655 (red) pre-treated tumours from day 8. E. Ration between p65 and pSTAT1 staining in 656 macrophages in tumour pre-treated with DMSO (gray) or ROCKi (red) from day 8. F. 657 Experimental design of the co-culture. G-H. Quantification of MMTV-PyMT-GFP+ cell growth 658 by measuring the total GFP+ area as a readout over the course of 4 days. Cells were grown 659 with DMSO-Macrophages (grey) or ROCKi-macrophages (purple) from day $8(G)$ or day 14 $660(\mathrm{H})$ tumours and normalised to the growth of MMTV-PyMT cells alone (dash line). Error bars represent the + SEM from three independent experiments. Biological replicates within each experiment are indicated with black, green or red data points in the graph. No statistically significant changes were observed between groups following student's t-test analysis.

664

665

666

667 


\section{Material and Methods}

\section{Statistical analysis}

670 Unless otherwise stated, statistical analyses were performed using Prism v.7.0c 671 (GraphPad Software). For column bar or scatter dot plots, error bars are the average $672 \pm$ Standard error of the mean (SEM). Boxplots show min to max values or 10-90 673 percentile. $P$ values were obtained by Student $t$-tests with paired or unpaired 674 samples when analysing the differences in one variable between two experimental groups. Two-way ANOVA was used where indicated to perform multiple variable analyses or multiple comparisons between experimental groups. Significance was set at $P<0.05$ and either the actual $P$ value or symbols describing it $\left({ }^{*}, p<0.05 ;{ }^{* *}\right.$, $\left.p<0.01 ;{ }^{* \star *}, p<0.001 ;{ }^{* \star * *}, p<0.0001\right)$ are displayed in graphs.

Data were pooled from at least two independent experiments and the exceptions are specified in the respective figure legends. $n$ values represent biological replicates, with the exception of the 3D Alvetex scaffold assays, for which both technical and biological replicates are shown.

\section{Bioinformatic analysis}

Gene expression data were analysed by the Bioinformatics \& Biostatistics facility at the Francis Crick Institute.

Biological replicate libraries were prepared using the polyA KAPA mRNA HyperPrep Kit and sequenced on Illumina HiSeq 4000 platform, generating 25 million 76bp single-end reads per sample. Read quality trimming and adaptor removal was carried out using Trimmomatic (version 0.36) (Bolger et al. 2014). The RSEM package (version 1.3.30) (Li \& Dewey 2011) in conjunction with the STAR alignment algorithm (version 2.5.2a) (Dobin et al. 2012) was used for the mapping and subsequent gene-level counting of the sequenced reads with respect to Ensembl mouse GRCm.38.89 version transcriptome. All parameters were run as default except "-forward-prob" which was set to 0.5. Normalisation of raw count data and differential expression analysis was performed with the DESeq2 package (version 1.18.1) (Love et al. 2014) within the R programming environment (version 3.4.3) (Team 2008). Differentially expressed genes were defined as those showing statistically significant differences (FDR $<0.05$ ) in the ROCKi group relative to the Control Group for both Fibroblast and Macrophage datasets. To determine pathway and biological process enrichment, Clarivate Analytics's Metacore pathway analysis tools was used to determine pathway and biological process enrichment using a hypergeometric test. [https://portal.genego.com/]. Gene lists ranked by the Wald statistic were used to look for pathway and selected genesets using the Broad's GSEA software (version 2.1.0) with genesets from MSigDB (version 6) (Subramanian et al. 2005).

Differentially regulated genes $(\log F C>1$ and FDR $<0.05)$ from the fibroblast data analysis were converted to human orthologs using HomoloGene [https://www.ncbi.nlm.nih.gov/homologene]. Breast Carcinoma (BRCA) normalised expression data was downloaded from the FireHose Broad GDAC website [https://gdac.broadinstitute.org/] Data downloads were all version 2016012800.0.0. BRCA patients were ranked using the sum of expression values of down regulated gene signature. The top quartile of patients were names as "HIGH", and the bottom quartile named "LOW". Survival plots were generated using R's survival package 
bioRxiv preprint doi: https://doi.org/10.1101/2021.06.15.448466; this version posted June 15, 2021. The copyright holder for this preprint (which was not certified by peer review) is the author/funder. All rights reserved. No reuse allowed without permission.

717 (version 2.41-3) (Therneau 2015) and the level of statistical significance determined

718 using the log rank test.

\section{Animal procedures}

Mice used were: Wildtype mice in BALB/CJ background, MMTV-PyMT+, MMTVPyMT+ actin GFP mice and Rag1-/- in pure genetic FVB/N background and. Animals were maintained under specific pathogen-free conditions and handled in accordance with the Institutional Committees on Animal Welfare of the UK Home Office and all the procedure were performed under the PPL P83B37B3C licence.

725

726

727

\section{Cell lines}

728

729

730

731

732

733

734

735

736

737

738

739

740

741

742

743

744

745

746

747

748

749

750

751

752

753

754

755

756

757

758

759

760

761

762

763

764

This study used a variety of breast cancer cell lines derived from the mouse normal breast fibroblasts (mNBF) were a gift from E. Sahai and MMTV-PyMT cells were isolated from MMTV-PyMT tumours (FVB/N background) as previously described (Malanchi et al. 2011). The 4T1 and 4T07cell lines were provided by the Cell Services Unit of The Francis Crick Institute.

4T1 cells plated in 2D were treated with either vehicle (DMSO) or $5 \mu \mathrm{M}$ of GSK269962A (Axon, 1167) for five days before performing in vitro and in vivo experiments, unless stated differently. Media and inhibitors were changed daily.

Matrices of 2:1 Pure Collagen-I and Matrigel were prepared to a final collagen concentration of $\sim 4 \mathrm{mg} / \mathrm{ml}$ and a Matrigel concentration of $\sim 2 \mathrm{mg} / \mathrm{ml}$. The cells were seeded on top in 200ul of growth medium.

\section{Generation of shRNA plasmids}

The doxycycline inducible Tet-PLKO-puro lentiviral plasmid vector used in this study was purchased from Addgene (\#21915, \#21916). The vector contains all the necessary components for the controlled doxycycline-triggered expression of shRNA in mammalian cells. Gene knock-down is triggered by the presence of doxycycline in the growth media, while in the absence of doxycycline, shRNA expression is blocked by the presence of constitutively-expressed TetR protein.

Forward and reverse oligonucleotides for a MYL9 and MYL12b-targeting shRNA and a nontargeting shRNA construct were designed using The RNAi Consortium collection (MISSION® shRNA, Sigma Aldrich).

\section{MACS isolation of cell subsets from tumour}

Tumour tissues were dissociated and processed to single-cells as described previously (Malanchi et al. 2011). MidiMACS separators and LD and LS columns (Milteny, 130-042-901, 130-042-401) were used to magnetically isolate mouse cellular subsets from tumour tissue at day 8 and day 14 and the procedure was performed on ice and promptly.

For fibroblast isolation, cells were isolated from Balb/cAnN mice orthotopically transplanted with $4 \mathrm{~T} 1$ tumours pre-treated with DMSO or GSK. Single-cell suspensions were incubated with mouse FcR Blocking Reagent and subsequently with the following APC labelled antibodies - CD45, CD31, CD326 and hCD2. Cells were washed and incubated with magnetic anti-APC MicroBeads (Miltenyi, 130-090- 
bioRxiv preprint doi: https://doi.org/10.1101/2021.06.15.448466; this version posted June 15, 2021. The copyright holder for this preprint (which was not certified by peer review) is the author/funder. All rights reserved. No reuse allowed without permission.

855). Magnetically labelled immune, endothelial, epithelial and cancer cells were isolated using LD columns, washed thrice with $3 \mathrm{ml}$ of MACS and the unlabelled flow-through cell fraction was the mesenchymal cells that we will refer to as fibroblasts hereafter. To increase the purity of the cells, the eluted fraction was applied to a second round of separation with a new LD column.

For macrophage isolation, $\mathrm{F} 4 / 80+$ cells were obtained by using the Anti-F4/80 MicroBeads UltraPure kit (Milteny, 130-110-443) following the manufacturer's instructions. Approximately $2 \times 10^{7}$ single-cells from the tumour were stained with a mix of $180 \mu \mathrm{l}$ MACS buffer and $20 \mu \mathrm{l}$ of Anti-F4/80 MicroBeads for 15 minutes at $4^{\circ} \mathrm{C}$ in the dark. During this incubation, an LS column was placed onto a MidiMACS separator where magnetic field was applied, and the column was rinsed with $3 \mathrm{ml}$ of MACS buffer. Cells were washed twice with $4 \mathrm{ml}$ of MACS buffer and resuspended in $1 \mathrm{ml}$ prior to proceeding to magnetic separation by applying the cell suspension onto the column. Columns were washed thrice with $3 \mathrm{ml}$ of MACS buffer before being carefully removed from the separator and placed on a FACS tube. $4.5 \mathrm{ml}$ of MACS buffer was added onto the LS column and the plunger was firmly pushed to elute the F4/80+ cell fraction which had been retained within the column due to the $\mathrm{F} 4 / 80$ + positive selection. To increase the purity of the cells, the eluted fraction was applied to a second round of separation with a new LS column.

\section{Alvetex scaffold 3D assay}

Alvetex Scaffold 96-well plates (ReproCELL, AVP009) were washed once with $70 \%$ ethanol to render it hydrophilic. Following three washes with PBS, they were coated with a collagen-solution and incubated for 1 hour at $37^{\circ} \mathrm{C}$ prior to cell seeding. Primary MMTV-PyMT actin-GFP cells were seeded at a density of 5,000 cells per well and fibroblasts and macrophages isolated from tumours were seeded at a density of 25,000 cells per well in a total volume of $100 \mathrm{ul}$ of complete MMTV-PyMT media.

The growth of GFP+ cells was monitored every two days using the SteREO LumarV12 stereomicroscope (Zeiss) and for a total of 6 days. Images were taken at a magnification of $19.4 X, Z+0.09$, exposed for 2 seconds and quantified using ImageJ software. For quantification, images were stacked and the Li's minimum cross entropy thresholding algorithm was used. All data was normalized to the growth of PyMT-GFP cells at each timepoints.

\section{Generation of conditioned media}

$100,0004 T 1$ cells (control or ROCKi treated) were plated on tissue culture treated 24-well plates in $1 \mathrm{ml}$ of complete DMEM media, which was conditioned at $37^{\circ} \mathrm{C}$ for $48 \mathrm{~h}$. The medium preparation was collected and spun at $1200 \mathrm{rpm}$ for $20 \mathrm{~min}$. The supernatant after this centrifugation was collected and used as conditioned media (CM).

\section{Fibroblast contraction assay}

To assess fibroblast activation via their ability to remodel an extracellular matrix, $7,5 \times 10_{4} \mathrm{mNBF}$ cells were embedded in $100 \mu \mathrm{l}$ of 2:1 collagen-I:Matrigel and seeded on a 35-mm glass-bottom dish (MatTek, P35-1.5-14-C). Once the gel had polymerized, a low height co-culture insert (Millipore, PICM-ORG-50) was placed on the dish and $1,5 \times 10_{5}$ tumour cells were seeded on the insert in $1 \mathrm{ml}$ of complete 
814 DMEM media. Gel contraction was monitored daily by taking photographs of the gels. The gel contraction value refers to the contraction observed after 3 days, which was measured using ImageJ software and calculated as the gel area in arbitrary units (A.U.).

\section{Cell proliferation assays - incucyte}

821

822

823

824

825

826

827

828

Cells were plated sub-confluently with numbers ranging from 1,000 to 10,000 cells per well into 96-well high content imaging black plates (Corning, CLS4580). Cell growth was monitored over 4-6 days using IncuCyteZOOM® Live Cell Imaging. All conditions were performed in triplicate and 4 images per well were acquired every 3 hours. The total area covered by cells was automatically measured by the software through confluency masks. The percentage of confluency over time was calculated as the average area covered by cells relative to the total well area.

\section{Cell migration assays}

829

830

831

832

833

834

835

836

837

838

839

840

841

842

Chemotaxis of mNBF fibroblasts was assessed using Incucyte Clearview 96-well chemotaxis plate with, $2 \%$ pore density following the manufacturer's protocol (Essen Bioscience, 4582). 1,000 mNBF cells in DMEM with 1\% FBS were loaded in the upper chamber compartment. CM from 4T1 DMSO or GSK was loaded in the lower chamber. Migration was monitored using the IncuCyteZOOM® Live Cell Imaging, and images of the upper and lower chambers were taken every hour. The total area covered by cells was automatically measured by image segmentation using the integrated chemotaxis analysis plugin.

\section{Flow cytometry (FACS)}

Prepared single-cell suspensions of mouse tissues and in vitro treated cancer cells were incubated with mouse FcR Blocking Reagent (Miltenyi, 130-092-575) for 10 min at $4^{\circ} \mathrm{C}$ followed by a 30 minute incubation at $4^{\circ} \mathrm{C}$ in the dark with a combination of the pre-labelled antibodies. Following two washes with MACS buffer, dead cells were stained with DAPI (4,6-Diamidino-2-phenylindole dihydrochloride, Sigma, D9542) and analysed with an LSRFortessa cell analyser running FACSDiva software (BD Biosciences) and FlowJo 10.4 .2 software (FlowJO, LCC 2006-2018). All cellsorting experiments were carried out using a BD Influx cell sorter (BD Biosciences).

849 Details of the primary antibodies used for immunohistochemistry in this study are 850 listed below.

851

852

Immunofluorescence

\begin{tabular}{|l|l|l|l|l|l|}
\hline Antigen & Source Ig & $\begin{array}{l}\text { Reactivity } \\
\text { against }\end{array}$ & Dilution & $\begin{array}{l}\text { Antigen } \\
\text { retrieval }\end{array}$ & $\begin{array}{l}\text { Catalogue } \\
\text { number }\end{array}$ \\
\hline SMA & Mouse & $\begin{array}{l}\text { Mouse, } \\
\text { Human }\end{array}$ & $1: 750$ & Citrate & $\begin{array}{l}\text { DAKO, } \\
\text { M0851 }\end{array}$ \\
\hline Endomucin & Rat & Mouse & & Citrate & sc-65495 \\
\hline Collagen IV & Rabbit & Mouse & $1: 500$ & Citrate & $\begin{array}{l}\text { USBiological } \\
\text { C7510-50B }\end{array}$ \\
\hline F4/80 & Rat & Mouse & $1: 100$ & Trypsin & $\begin{array}{l}\text { eBioscience, } \\
14-4801\end{array}$ \\
\hline pSer19- & Rabbit & Mouse, & $1: 50$ & Access & CST, 3671L \\
\hline
\end{tabular}




\begin{tabular}{|l|l|l|l|l|l|}
\hline MLC2 & polyclonal & Human & & $\begin{array}{l}\text { super } \\
\text { buffer }\end{array}$ & \\
\hline
\end{tabular}

854 FACS

\begin{tabular}{|c|c|c|c|c|c|}
\hline Antigen & Reacting Ig & Clone & Company & Dilution & $\begin{array}{l}\text { Catalogue } \\
\text { number }\end{array}$ \\
\hline CD45-AF780 & Mouse & 30-F11 & eBioscience & $1: 200$ & $47-0451-82$ \\
\hline CD45-bv421 & Mouse & $30-\mathrm{F} 11$ & Biolegend & $1: 200$ & 103133 \\
\hline CD45-APC & Mouse & $30-\mathrm{F} 11$ & eBioscience & $1: 200$ & $17-0451-83$ \\
\hline CD11b-AF780 & Mouse & $\mathrm{M} 1 / 70$ & eBioscience & $1: 100$ & $47-0112-82$ \\
\hline CD11b-APC & Mouse & $\mathrm{M} 1 / 70$ & Biolegend & $1: 100$ & 101212 \\
\hline F4/80-647 & Mouse & BM8 & eBioscience & $1: 100$ & MF48021 \\
\hline F4/80-AF780 & Mouse & BM8 & eBioscience & $1: 100$ & 47-4801-82 \\
\hline CD31-PECy7 & Mouse & 390 & eBioscience & $1: 100$ & $25-0311-82$ \\
\hline CD31-bv421 & Mouse & 390 & Biolegend & $1: 100$ & 102423 \\
\hline CD31-APC & Mouse & 390 & eBioscience & $1: 100$ & $17-0311-82$ \\
\hline hCD2-APC & Human & $\begin{array}{l}\text { RPA- } \\
2.10\end{array}$ & eBioscience & $1: 200$ & $17-0029-42$ \\
\hline CD19-bv421 & Mouse & 6D5 & Biolegend & $1: 100$ & 115549 \\
\hline CD11c-PE & Mouse & N418 & eBioscience & $1: 100$ & $12-0114-83$ \\
\hline $\begin{array}{l}\text { CD49f-PerCP- } \\
\text { eFluor710 }\end{array}$ & Mouse & $\begin{array}{l}\text { ebioGO } \\
\text { H3 }\end{array}$ & eBioscience & $1: 100$ & $46-0495-82$ \\
\hline EPCAM-APC & Mouse & G8.8 & eBioscience & $1: 100$ & $17-5791-81$ \\
\hline CD3e-AF780 & Mouse & 17A3 & eBioscience & $1: 200$ & $47-0032-80$ \\
\hline Ly6G-bv450 & Mouse & $1 \mathrm{~A} 8$ & eBioscience & $1: 200$ & 560603 \\
\hline Ly6G-PECy7 & Mouse & $1 \mathrm{~A} 8$ & Biolegend & $1: 100$ & 127618 \\
\hline gd TCR-PECy7 & Mouse & $\begin{array}{l}\text { eBioGL } \\
3\end{array}$ & eBioscience & $1: 100$ & $25-5711-82$ \\
\hline CD4-bv605 & Mouse & GK1.5 & Biolegend & $1: 100$ & 100451 \\
\hline CD8-PE & Mouse & $53-6.7$ & eBioscience & $1: 100$ & $12-0081-82$ \\
\hline CD163-PE & Mouse & $\begin{array}{l}\text { TNKUP } \\
\mathrm{J}\end{array}$ & Invitrogen & $1: 100$ & $12-1631-82$ \\
\hline MHCII-APC & Mouse & $\begin{array}{l}\text { M5/114 } \\
.15 .2\end{array}$ & Biolegend & $1: 100$ & 107613 \\
\hline CD86-PECy7 & Mouse & GL1 & eBioscience & $1: 100$ & $25-0862-82$ \\
\hline CD206-647 & Mouse & & $\begin{array}{l}\text { Life } \\
\text { technologies }\end{array}$ & $1: 100$ & $\begin{array}{l}\text { mca2235a64 } \\
7 t\end{array}$ \\
\hline Arg1-PECy7 & Mouse & A1exF5 & Invitrogen & $1: 100$ & $25-3697-82$ \\
\hline iNOS-PE & Mouse & CXNFT & Invitrogen & $1: 100$ & $12-5920-82$ \\
\hline
\end{tabular}




\section{Acknowledgments}

858 The graphic representation in the figures were created using BioRender.com.

859 We thank E. Sahai (The Francis Crick Institute) for scientific discussions. We thank Jose L.

860 Orgaz-Bueno, Eva Crosas-Molist, Irene Rodriguez-Hernandez, Stefania di Blasio, Victoria

861 Bridgeman and Emma Nolan for scientific discussions and support during the course of this

862 project. E. Nye from the Experimental Histopathology Unit at the Francis Crick Institute for

863 histological processing and analysis support; J. Bee from the Biological Resources Unit at

864 the Francis Crick Institute for technical support with mice and mouse tissues; R. Goldstone 865 and A. Edwards from the Advanced Sequencing Facility at the Francis Crick Institute for 866 technical support; the Flow Cytometry Unit at the Francis Crick Institute.

867 This work was supported by the Francis Crick Institute, which receives its core funding from 868 Cancer Research UK (FC001112), the UK Medical Research Council (FC001112), and the 869 Wellcome Trust (FC001112) and the European Research Council grant (ERC CoG-H2020870 725492). VSM lab was supported by Cancer Research UK (CRUK) C33043/A24478 and 871 Barts Charity.

872

873 Author contributions

874 A.P.R. designed and performed most of the experiments, analysed and interpreted the data 875 and contributed to the manuscript preparation. O.M. performed tumour staining and 876 quantification analysis of in vivo macrophages, performed secretome data analysis and 877 critically review the manuscript. L.O. assisted with some of the experimental design and 878 critically review the manuscript. P.C. performed bioinformatics analysis. I.M. and V.S.M. 879 designed and supervised the study, interpreted the data and wrote the manuscript. 880

\section{Conflict of interest}

882 The authors have no Conflict of interest.

883

\section{Data Availability}

885 The RNaseq datasets generated during this study are available will be made available at the 886 Gene Expression Omnibus and they can currently be provided to the reviewers upon 887 requests. 


\section{References}

890

891

892

893

894

895

896

897

898

899

900

901

902

903

904

905

906

907

908

909

910

911

912

913

914

915

916

917

918

919

920

921

922

923

924

925

926

927

Albrengues, J. et al., 2014. LIF mediates proinvasive activation of stromal fibroblasts in cancer. CellReports, 7(5), pp.1664-1678.

Bolger, A.M., Lohse, M. \& Usadel, B., 2014. Trimmomatic: a flexible trimmer for Illumina sequence data. Bioinformatics, 30(15), pp.2114-2120.

Costa, A. et al., 2018. Fibroblast Heterogeneity and Immunosuppressive Environment in Human Breast Cancer. CCELL, 33(3), pp.463-479.e10.

Coussens, L.M. \& Werb, Z., 2002. Inflammation and cancer. Nature, 420(6917), pp.860867.

del Pozo Martin, Y. et al., 2015. Mesenchymal Cancer Cell-Stroma Crosstalk Promotes Niche Activation, Epithelial Reversion, and Metastatic Colonization. CellReports, 22(13 (11)), pp.2456-2469.

Dobin, A. et al., 2012. STAR: ultrafast universal RNA-seq aligner. Bioinformatics, 29(1), pp.15-21.

Erez, N. et al., 2010. Cancer-Associated Fibroblasts Are Activated in Incipient Neoplasia to Orchestrate Tumor-Promoting Inflammation in an NF-\&kappa;B-Dependent Manner. CCELL, 17(2), pp.135-147.

Friedman, G. et al., 2020. Cancer-associated fibroblast compositions change with breast cancer progression linking the ratio of S100A4 + and PDPN + CAFs to clinical outcome. Nature Cancer, 1(7), pp.692-708.

Georgouli, M. et al., 2019. Regional Activation of Myosin II in Cancer Cells Drives Tumor Progression via a Secretory Cross-Talk with the Immune Microenvironment. Cell, 176(4), pp.757-774.e23.

Katlinski, K.V. et al., 2017. Inactivation of Interferon Receptor Promotes the Establishment of Immune Privileged Tumor Microenvironment. CCELL, 31(2), pp.194-207.

Kümper, S. et al., 2016. Rho-associated kinase (ROCK) function is essential for cell cycle progression, senescence and tumorigenesis. eLife, 5, pp.e12994-e12994.

Laklai, H. et al., 2016. Genotype tunes pancreatic ductal adenocarcinoma tissue tension to induce matricellular fibrosis and tumor progression. Nature Medicine, 22(5), pp.497505.

Li, B. \& Dewey, C.N., 2011. RSEM: accurate transcript quantification from RNA-Seq data with or without a reference genome. BMC bioinformatics, 12(1), p.323.

Love, M.I., Huber, W. \& Anders, S., 2014. Moderated estimation of fold change and dispersion for RNA-seq data with DESeq2. Genome biology, 15(12), p.550.

Malanchi, I. et al., 2011. Interactions between cancer stem cells and their niche govern metastatic colonization. Nature, 481(7379), pp.85-89.

Mantovani, A. et al., 2017. Tumour-associated macrophages as treatment targets in oncology. Nature Reviews Clinical Oncology, 144, p.2409. 
Northcott, J.M. et al., 2018. Feeling Stress: The Mechanics of Cancer Progression and Aggression. Frontiers in Cell and Developmental Biology, 6, p.1120.

Noy, R. \& Pollard, J.W., 2014. Tumor-associated macrophages: from mechanisms to therapy. Immunity, 41(1), pp.49-61.

Ogura, M., Bridgeman, V.L. \& Malanchi, I., 2017. Macrophages unlock progression of breast cancer cells experiencing matrigel-segregation in transplantation models. Scientific Reports, 7(1), p.1423.

Ombrato, L. et al., 2019. Metastatic-niche labelling reveals parenchymal cells with stem features. Nature, 572(7771), pp.603-608.

Orgaz, J.L. et al., 2020. Myosin II Reactivation and Cytoskeletal Remodeling as a Hallmark and a Vulnerability in Melanoma Therapy Resistance. CCELL, 37(1), pp.85-103.e9.

Östman, A. \& Augsten, M., 2009. Cancer-associated fibroblasts and tumor growth-bystanders turning into key players. Current Opinion in Genetics \& Development, 19(1),

941 pp.67-73.

Rodriguez-Hernandez, I. et al., 2016. Rho, ROCK and actomyosin contractility in metastasis as drug targets. F1000Research, 5, p.783.

Rodriguez-Hernandez, I. et al., 2020. WNT11-FZD7-DAAM1 signalling supports tumour initiating abilities and melanoma amoeboid invasion. Nature Communications, 11(1), pp.1-20.

946

Sanz-Moreno, V. et al., 2011. ROCK and JAK1 Signaling Cooperate to Control Actomyosin Contractility in Tumor Cells and Stroma. Cancer cell, 20(2), pp.229-245.

Subramanian, A. et al., 2005. Gene set enrichment analysis: a knowledge-based approach for interpreting genome-wide expression profiles. Proceedings of the National Academy of Sciences of the United States of America, 102(43), pp.15545-15550.

Team, T., 2008. The R Project for Statistical Computing. www.r-project.org.

Therneau, T.M., 2015. Survival Analysis [R package survival version 3.2-3]. Available at: https://CRAN.R-project.org/package=survival.

Van Rooijen, N. \& Hendrikx, E., 2010. Liposomes for specific depletion of macrophages from organs and tissues. Methods in molecular biology (Clifton, N.J.), 605, pp.189-203.

Vicente-Manzanares, M. et al., 2009. Non-muscle myosin II takes centre stage in cell adhesion and migration. Nature Reviews Molecular Cell Biology, 10(11), pp.778-790.

Wagner, J. et al., 2019. A Single-Cell Atlas of the Tumor and Immune Ecosystem of Human Breast Cancer. Cell, 177(5), pp.1330-1345.e18.

Wculek, S.K. \& Malanchi, I., 2015. Neutrophils support lung colonization of metastasisinitiating breast cancer cells. Nature, 17(528), pp.413-417. 
bioRxiv preprint doi: https://doi.org/10.1101/2021.06 15.448466; this version posted June 15, 2021. The copyright holder for this preprint (which was not certified by peer review) is the author/funder. All rights reserved. No reuse allowed without permission.

Figure 1

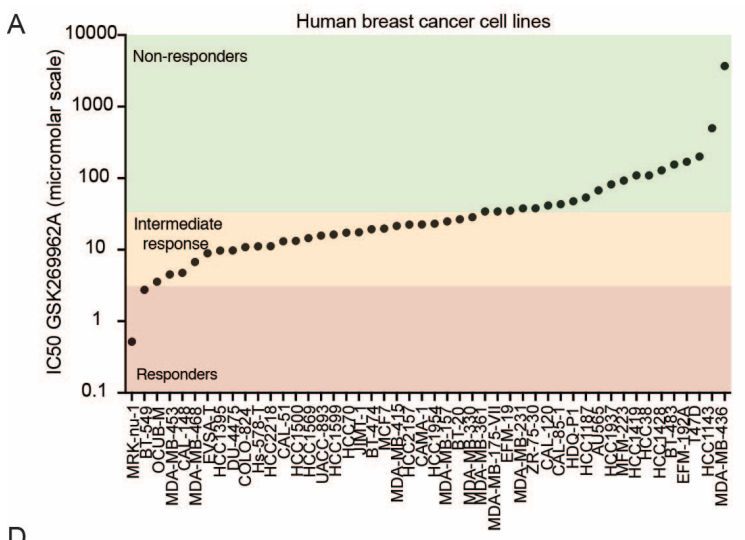

$$
\text { B }
$$
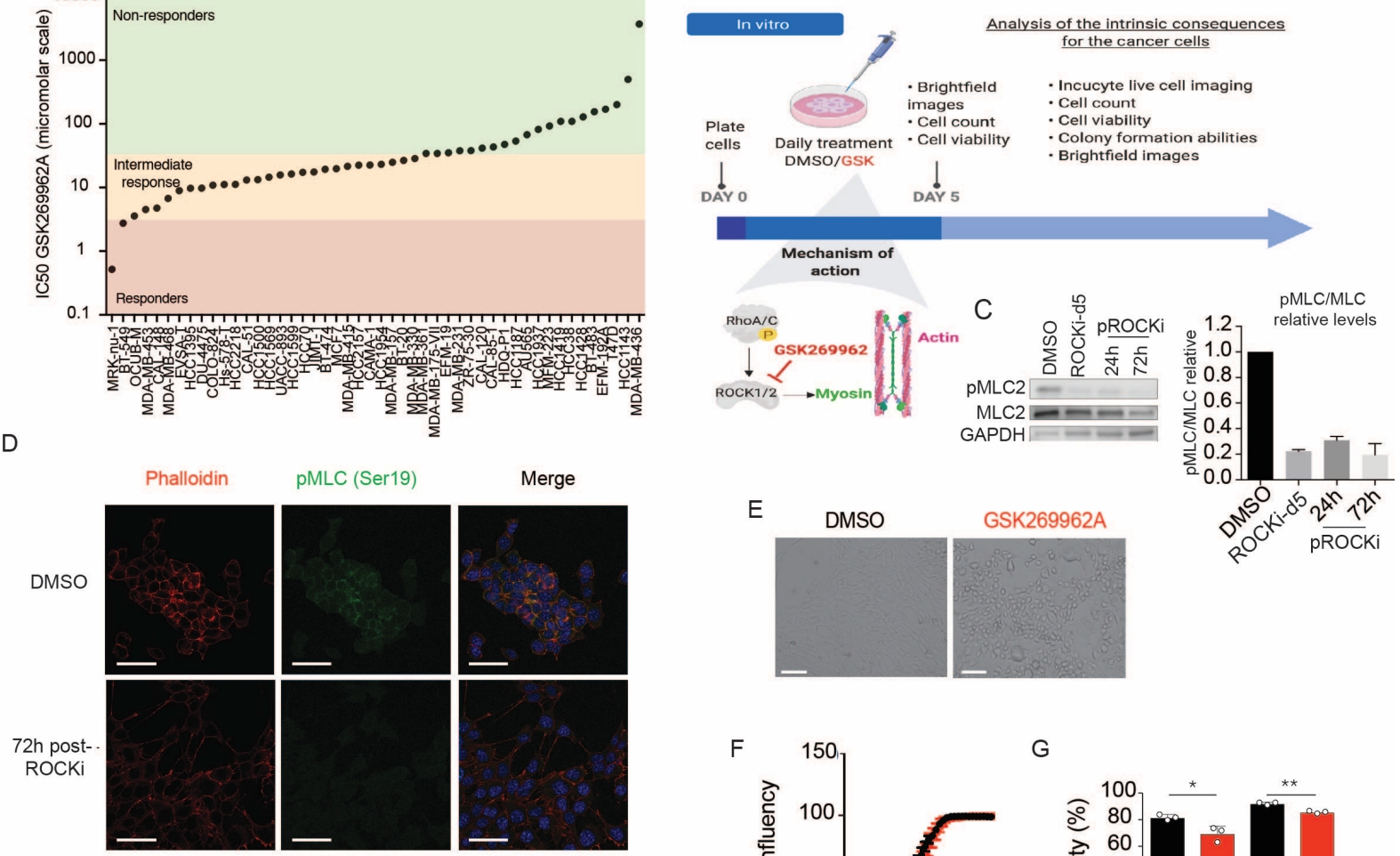

$\mathrm{H}$

Conditioned

F

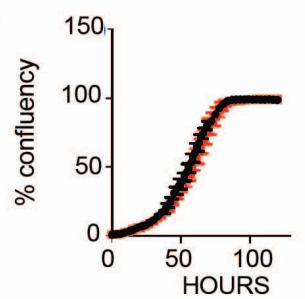

G

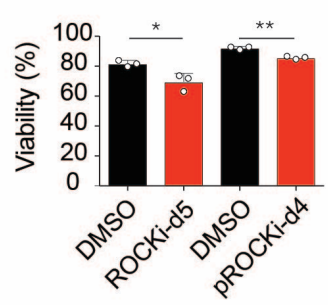

TOP hits

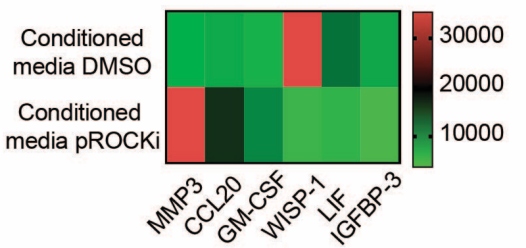


Figure 2

A

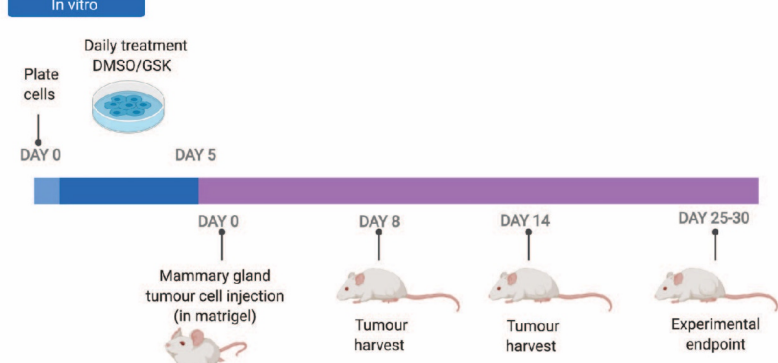

C

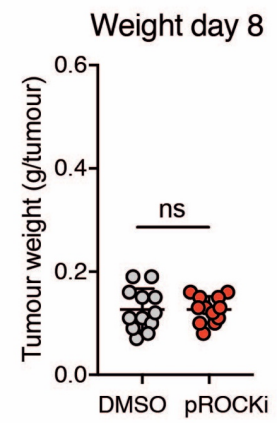

D

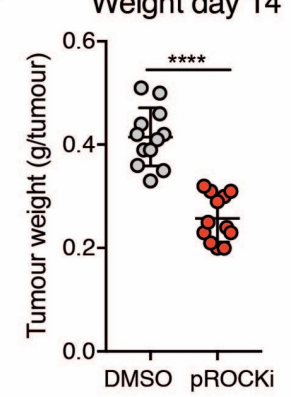

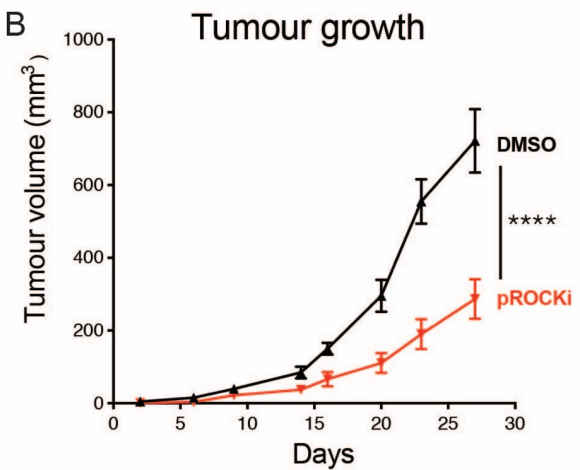

E pMLC2-early tumour growth

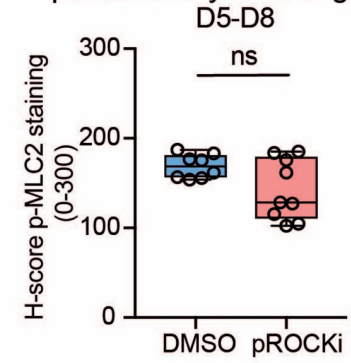

$\square \mathrm{DMSO}$

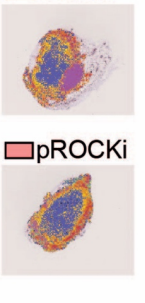

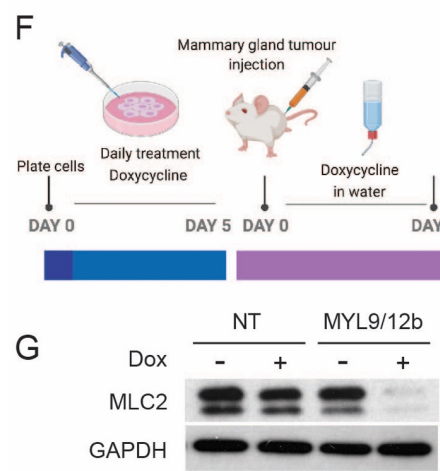

H Non-targeting shRNA MYL9/12b shRNA
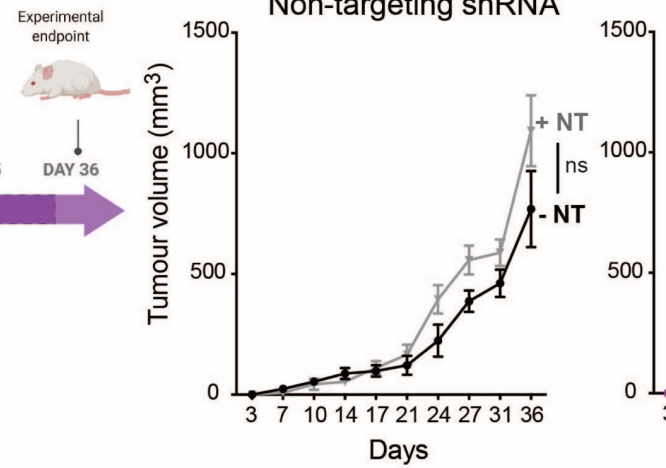

Days
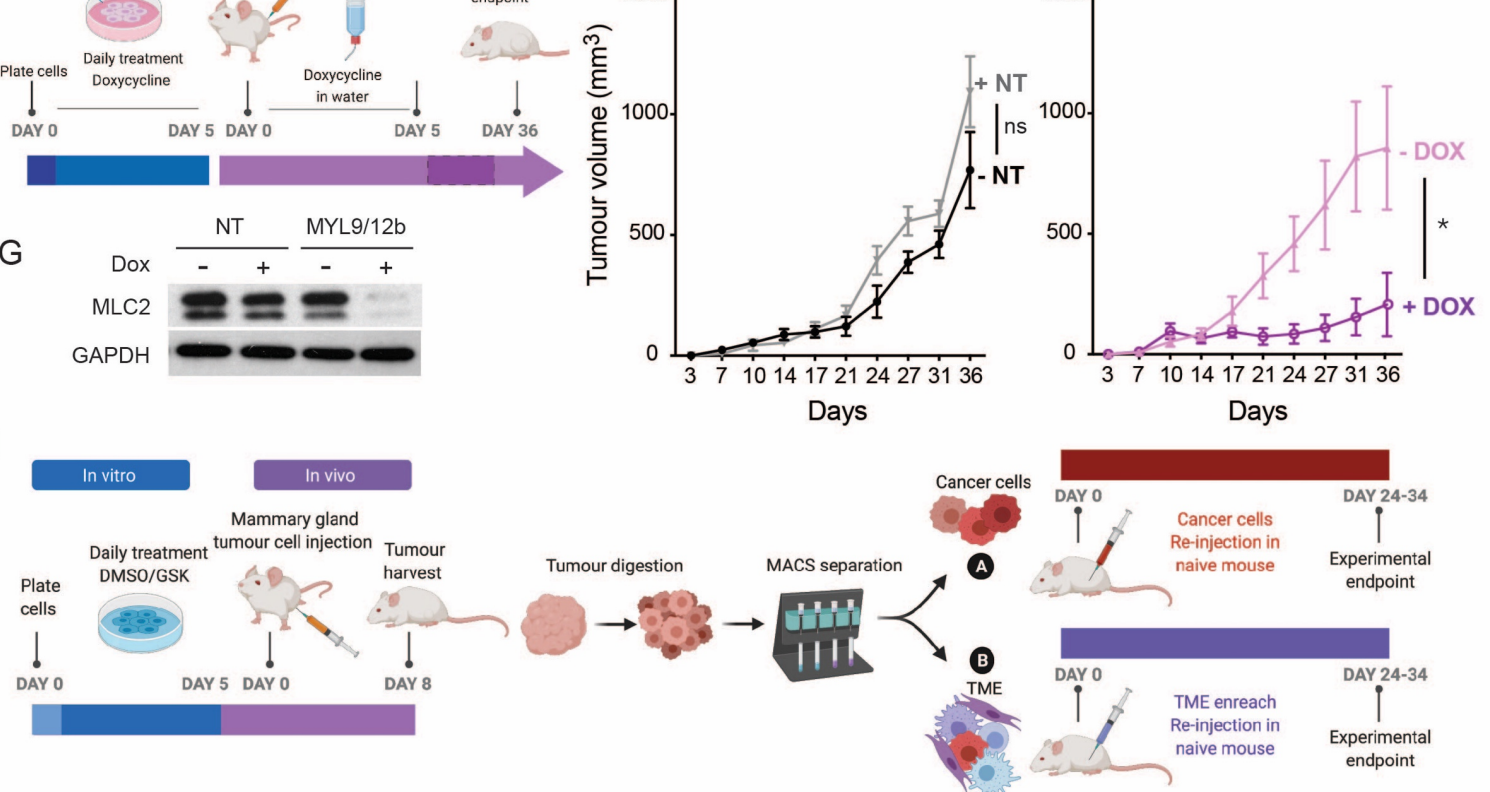

$\mathrm{J}$

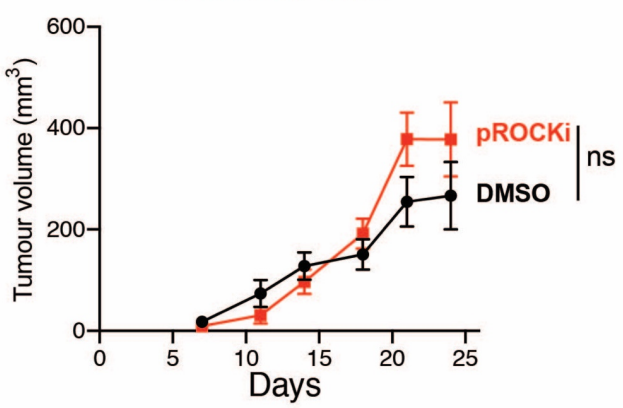

K $\quad$ B. Tumour microenvironment

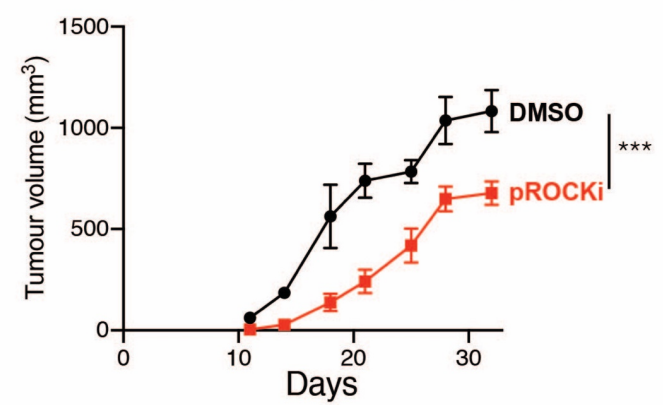


bioRxiv preprint doi: https://doi.org/10.1101/2021 06.15.448466; this version posted June 15, 2021. The copyright holder for this preprint (which was not certified by peer review) is the author/funder. All rights reserved. No reuse allowed without permission.

\section{Figure 3}

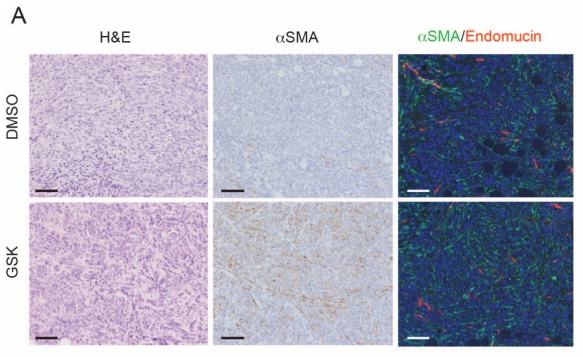

F
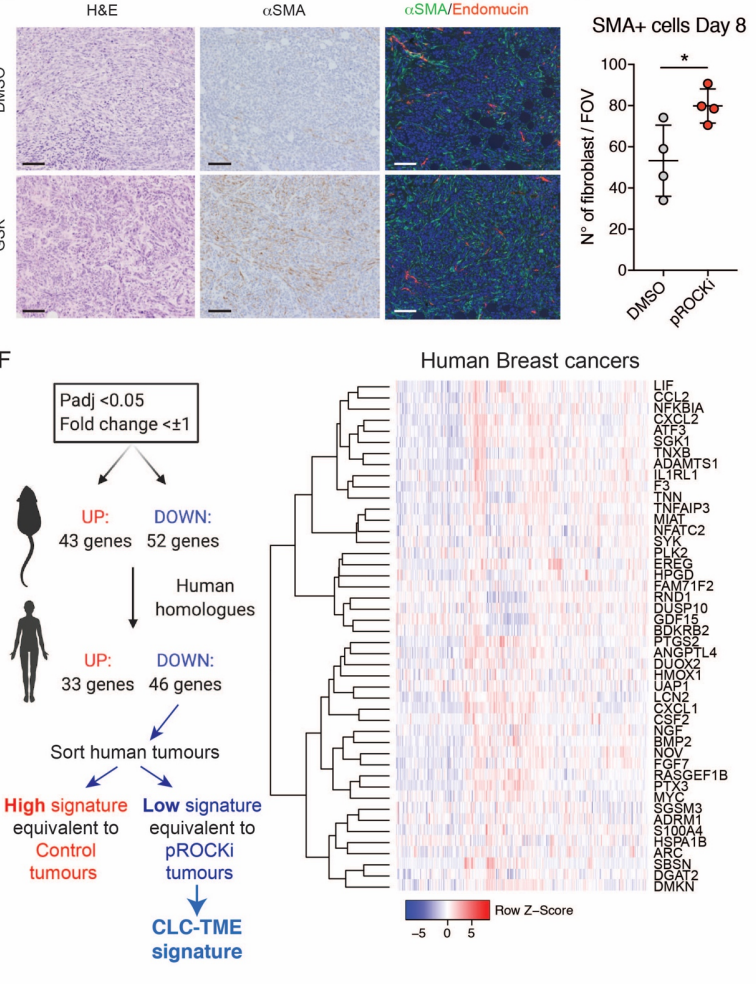

C

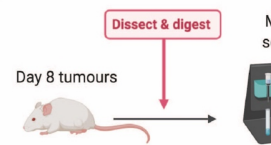

$\mathrm{D}$

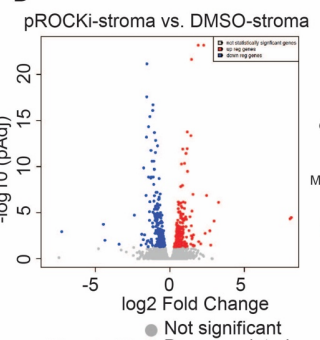

DOWNREGULATED UPREGULATED

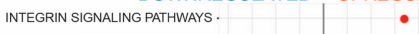

MATRIX REGULATION

GRONTH

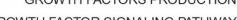

мотLITY

MOTLITY.

CORE SIGNALING

INFLAMMATORY SIGNALING PATHWAYS

Threshold Nownregulated

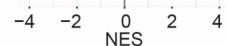

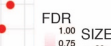

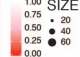
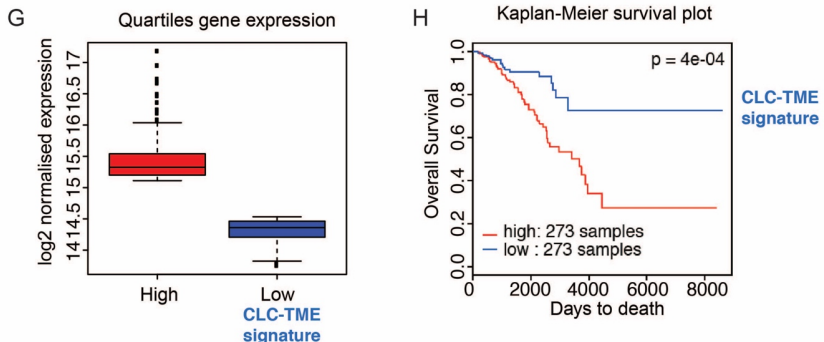
Figure 4
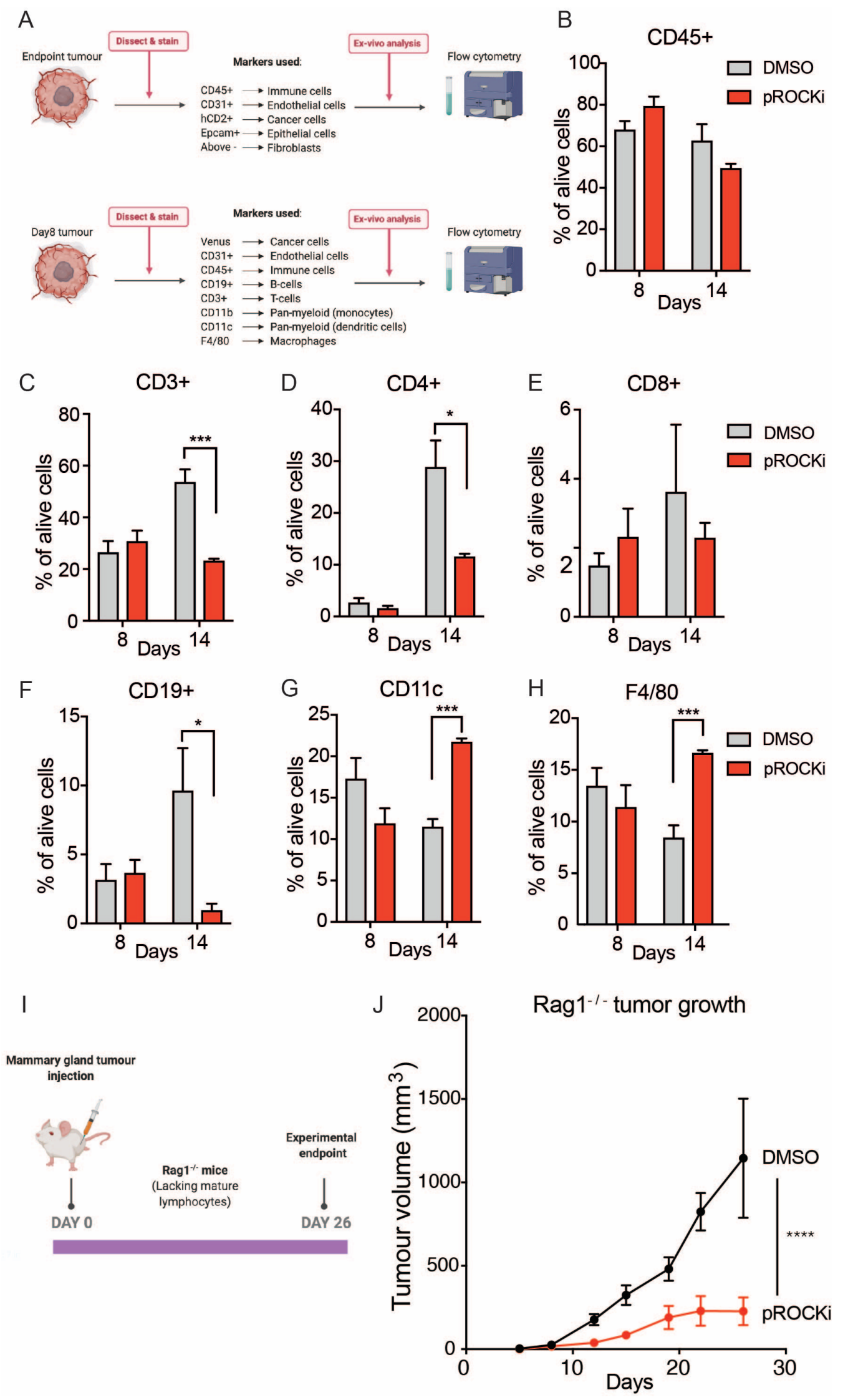
bioRxiv preprint doi: https://doi.org/10.1101/2021.06.15.448466; this version posted June 15, 2021. The copyright holder for this preprint (which was not certified by peer review) is the author/funder. All rights reserved. No reuse allowed without permission.

Figure 5

A
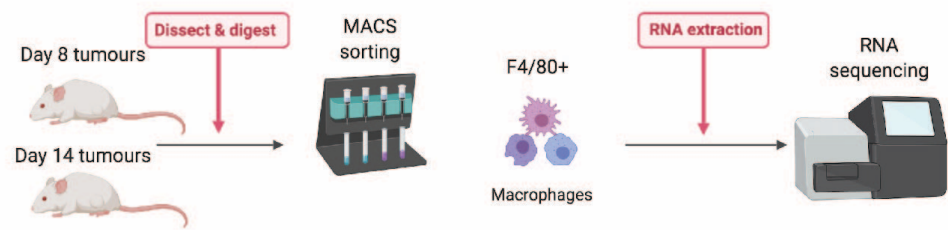

B

Heatmap iROCK d14 vs. DMSO d14

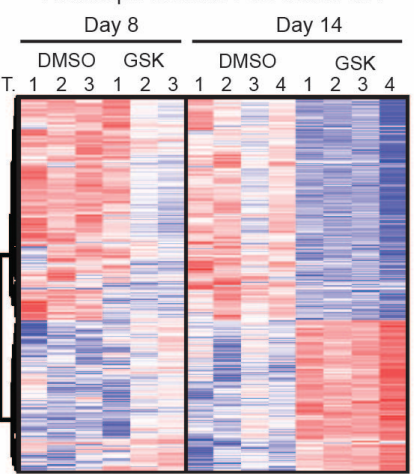

C IROCK day 8 vs DMSO day $8 \quad$ iROCK day 14 vs DMSO day 14

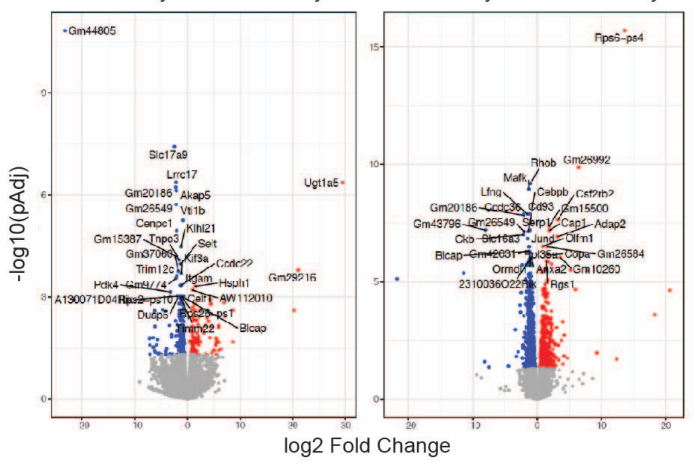

olcano plots

Threshold

Not significant

Downregulated

Upregulated

$$
\begin{array}{ll}
\text { Gene expression changes } & \text { Gene expression changes } \\
1692 \text { significant differences } & 336 \text { significant differences }
\end{array}
$$

D

Selected pathways enreached in pROCKi vs DMSO

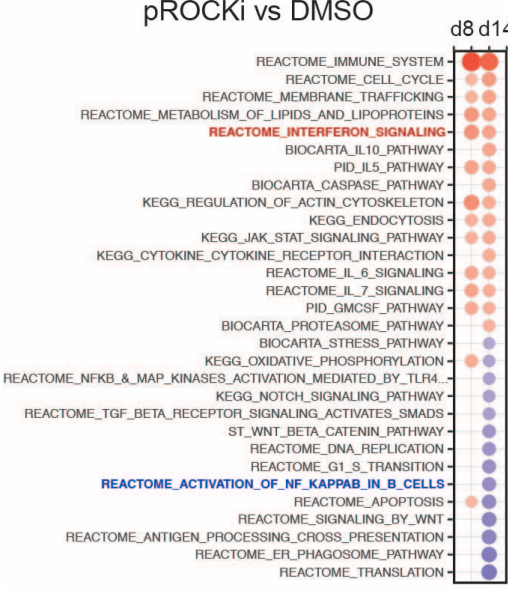

$\mathrm{H}$

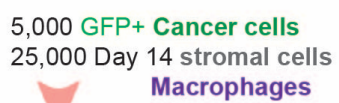

E PSTAT1+ Macrophages

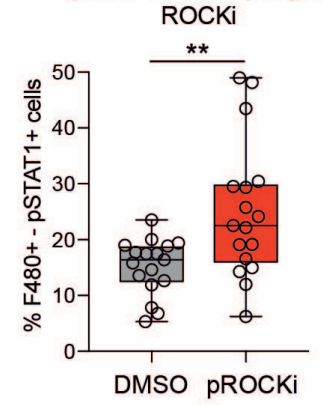

G pSTAT1+ Macrophages

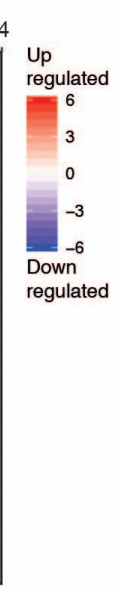

F
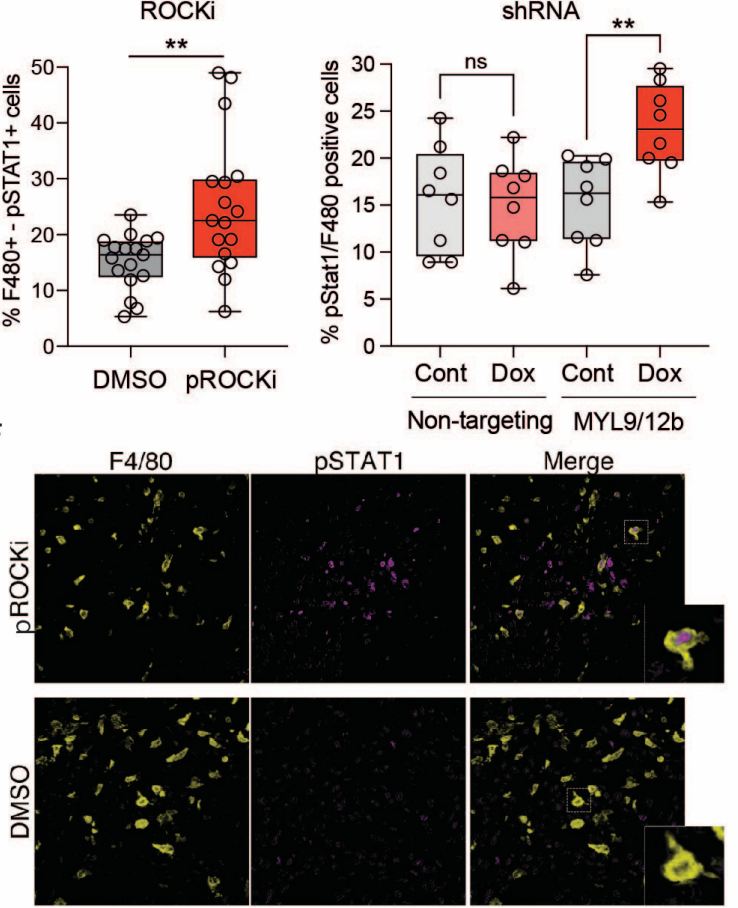

I $\square$ DMSO-TAM + CAF $\square$ pROCKI-TAM + CAF

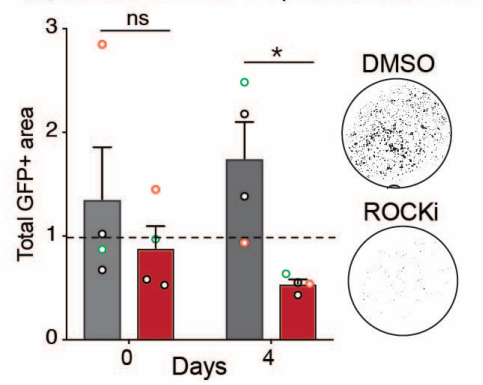

$J$

Growth of MMTV-PyMT cells

pROCKi-stroma + DMSO-TAM pROCKi-stroma + ROCKi TAM

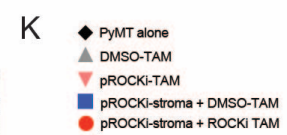

$\frac{2}{\frac{2}{2}}$

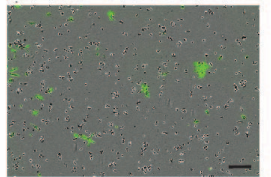

$\frac{0}{2}$

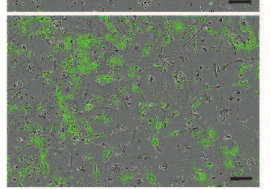


Figure 6

A

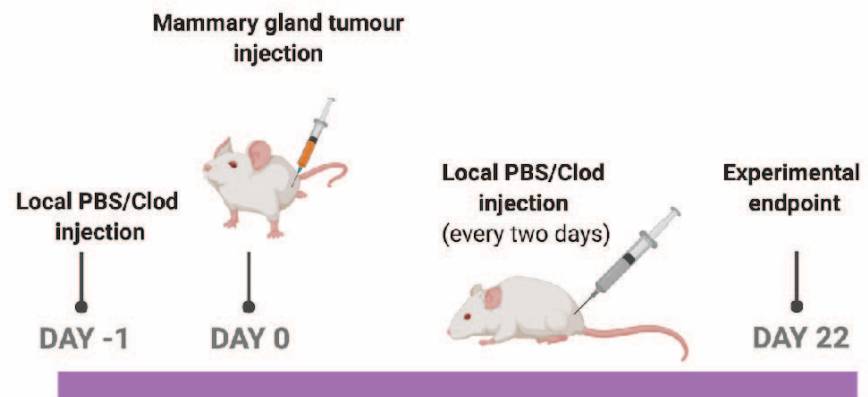

B

C
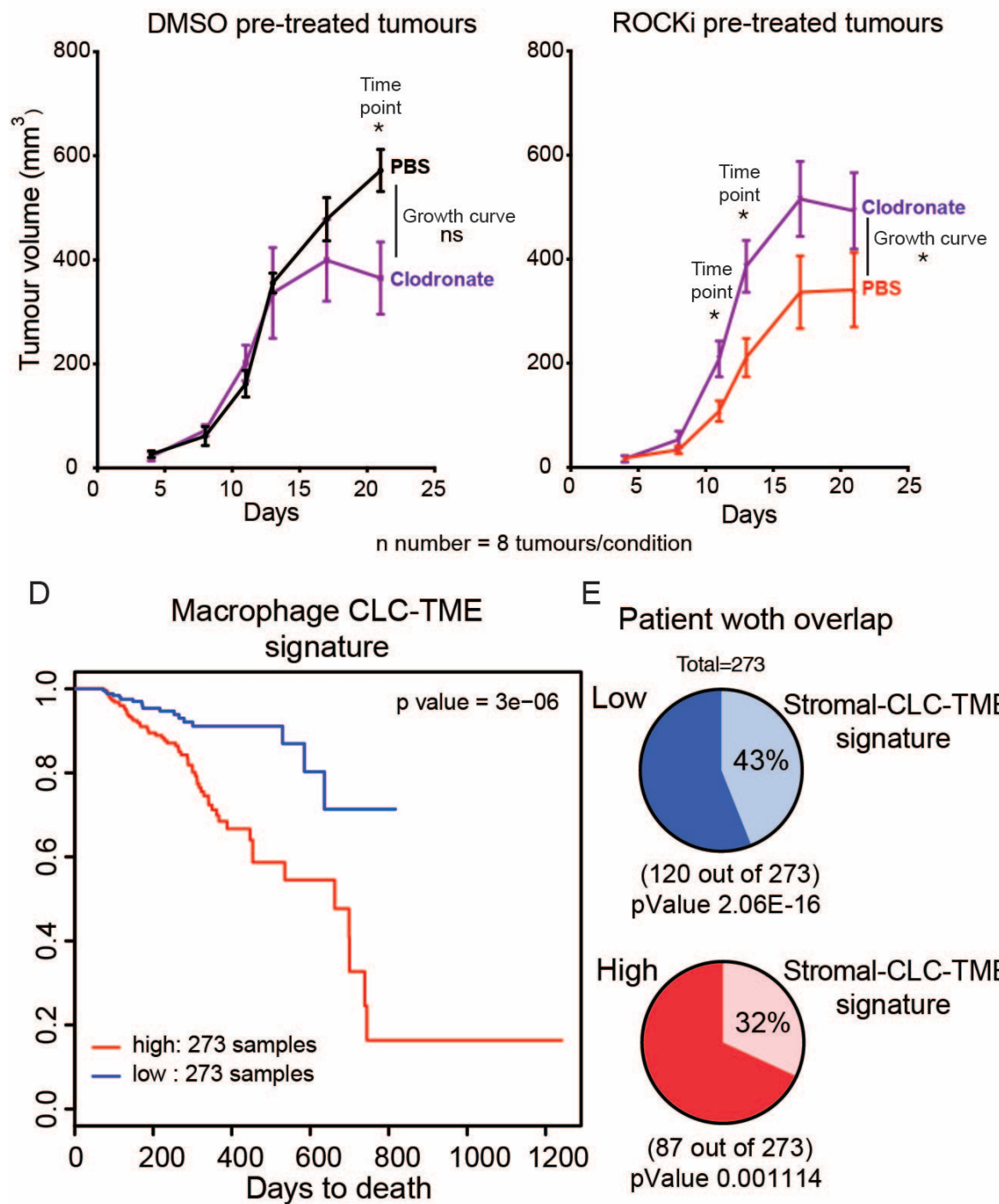

E Patient woth overlap
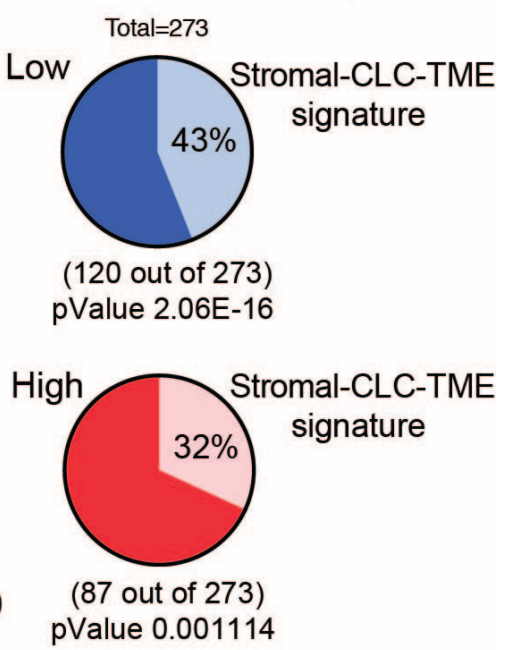
bioRxiv preprint doi: https://doi.org/10.1101/2021.06 15.448466; this version posted June 15, 2021. The copyright holder for this preprint (which was not certified by peer review) is the author/funder. All rights reserved. No reuse allowed without permission.

\section{Figure 7}

\section{Early stage}

Late stage

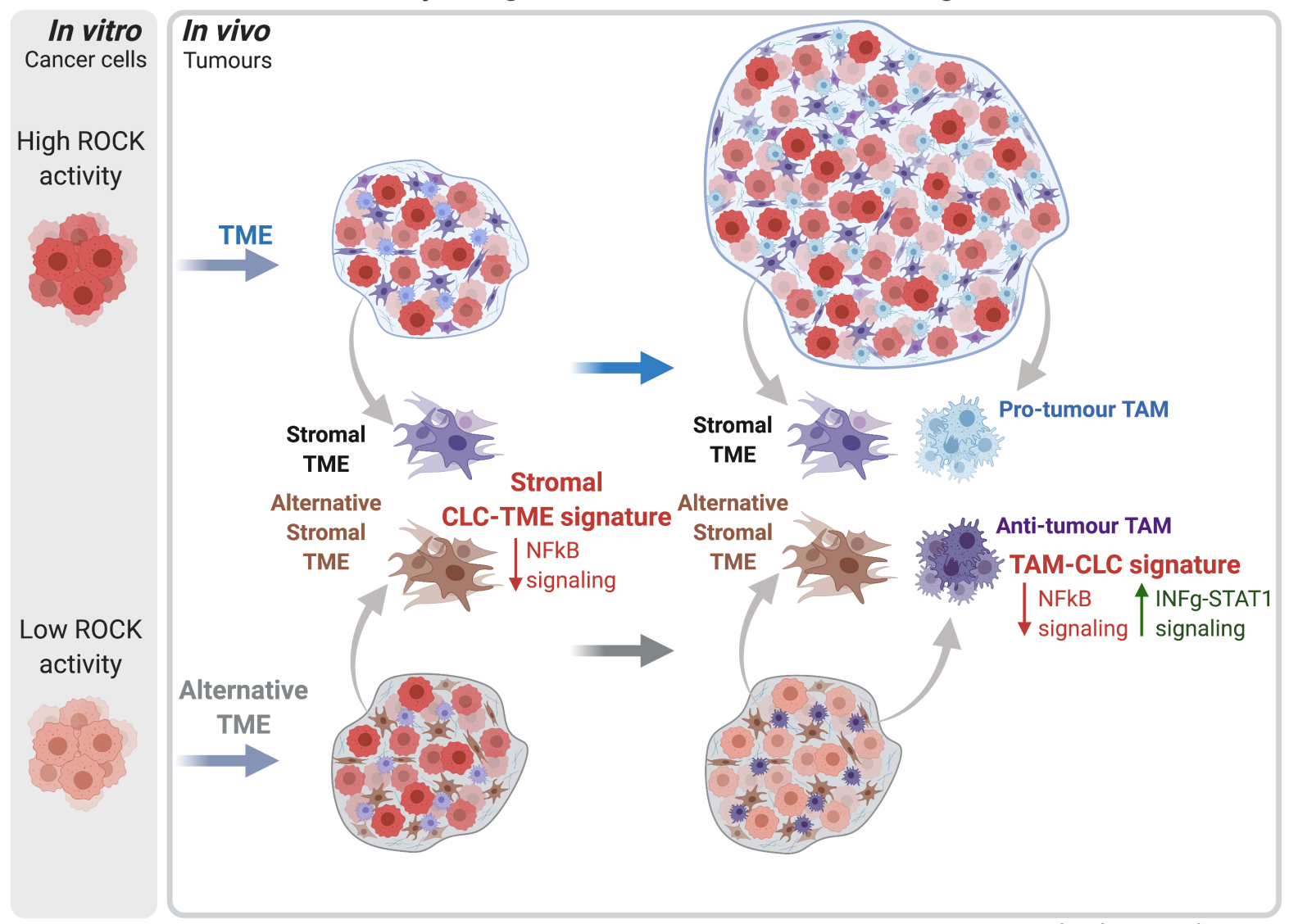

Created with BioRender.com 
bioRxiv preprint doi: https://doi.org/10.1101/2021.06 15.448466; this version posted June 15, 2021. The copyright holder for this preprint (which was not certified by peer review) is the author/funder. All rights reserved. No reuse allowed without permission.

\section{Supplementary Figure 1}

A

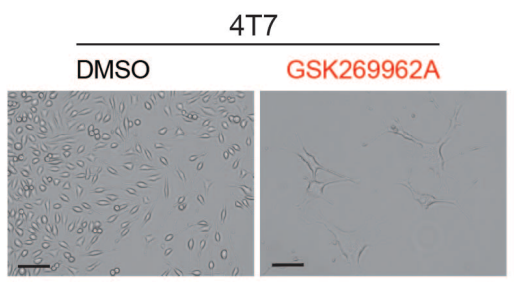

B

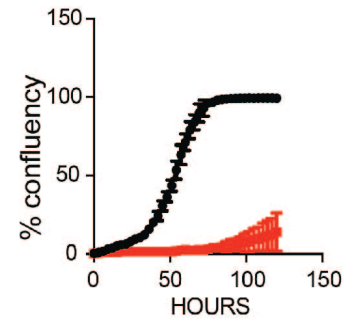

E

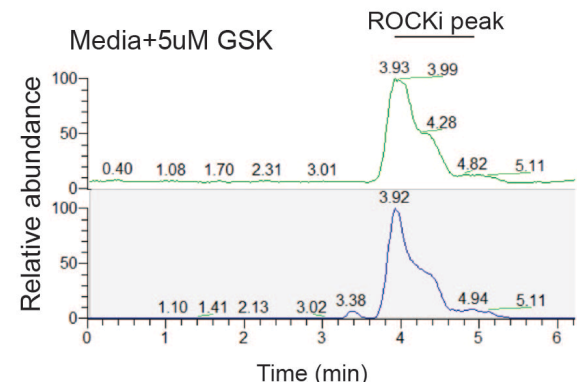

$\mathrm{F}$

Fibroblast proliferation

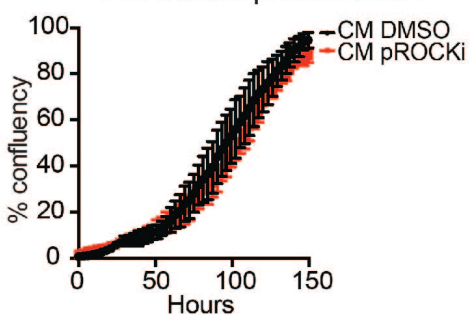

G

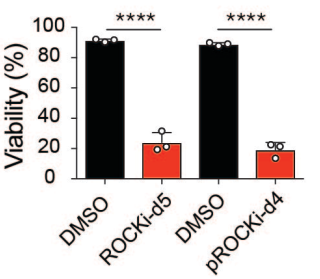

C

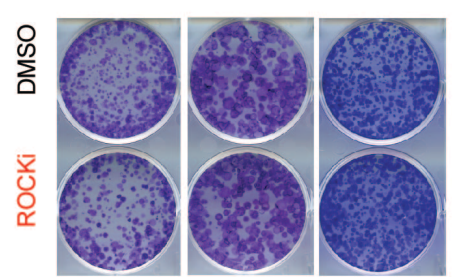

D Plastic

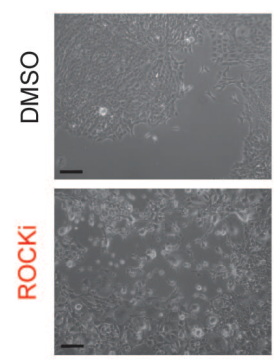

Collagen-Matrigel Embedded in matrigel
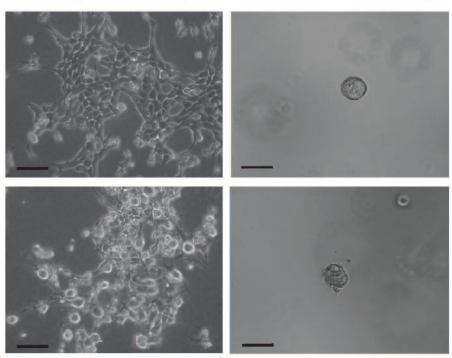

CM GSK

Base peak chromatogram in positive mode

Base peak chromatogram in negative mode

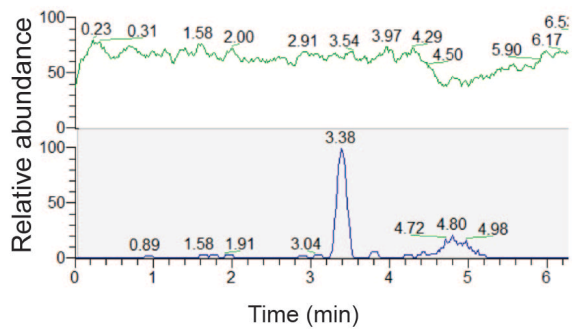

$\mathrm{H}$

Fibroblast Morphology

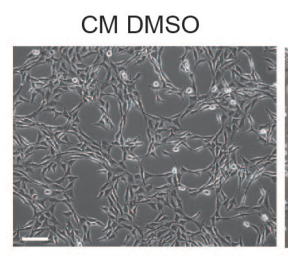

CM pROCKi
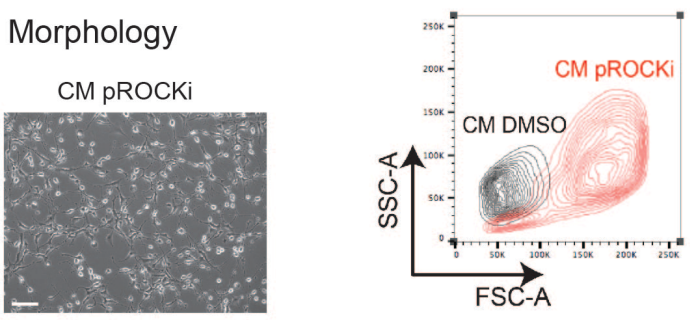

Fibroblast migration

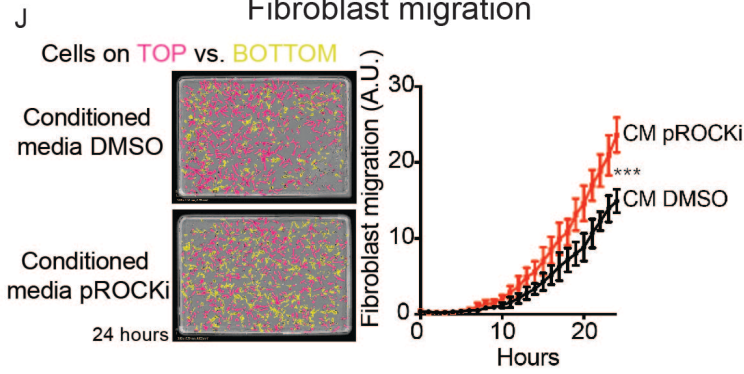




\section{Supplementary Figure 2}

A Day 8 tumours

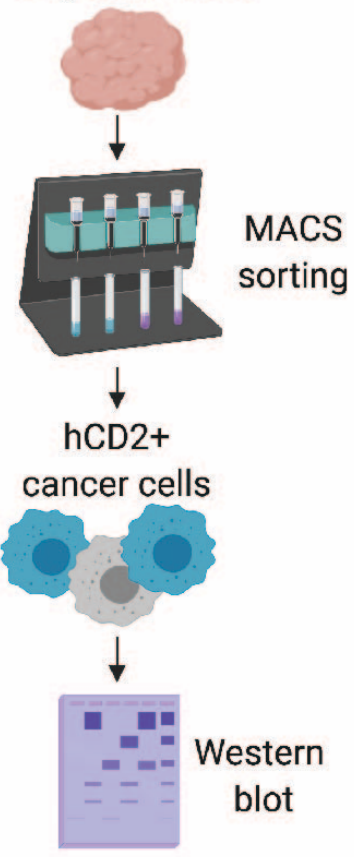

B
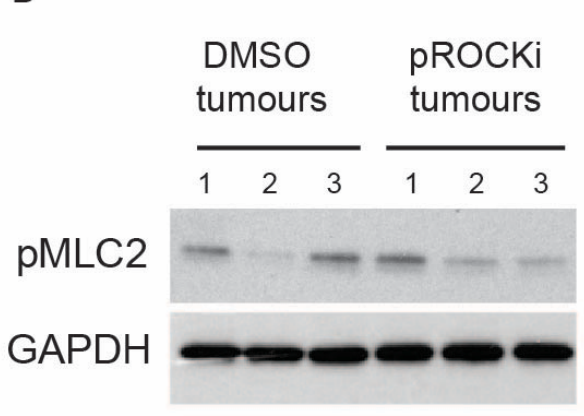

D

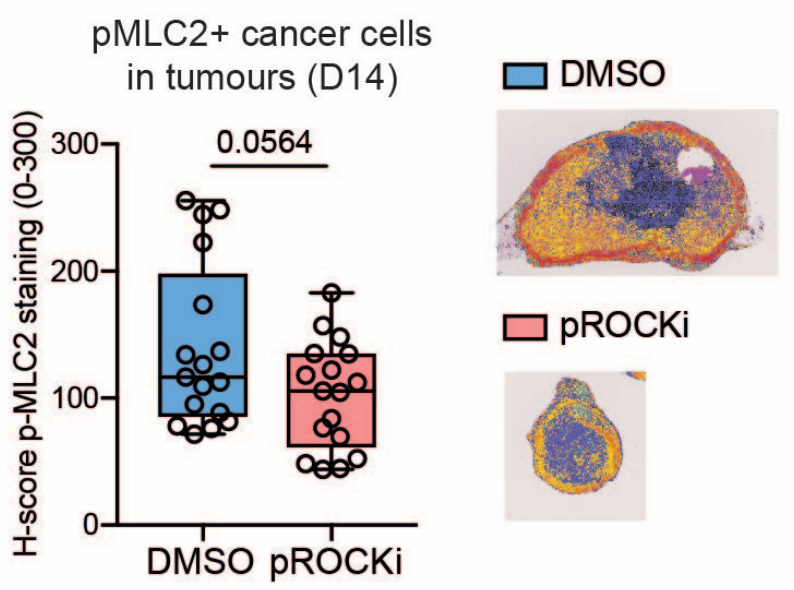

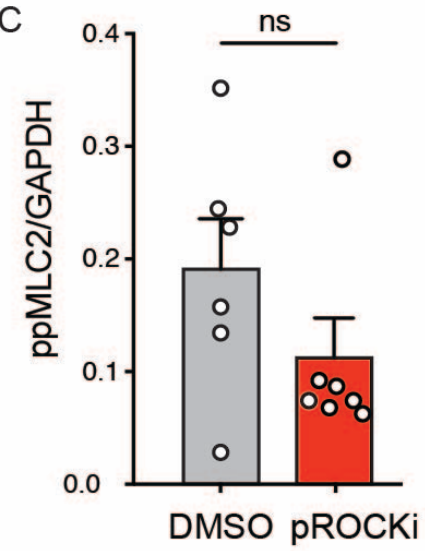


bioRxiv preprint doi: https//doi.org/10 1101/2021 06 15.448466; this version posted June 15, 2021. The copyright holder for this preprint (which was not certified by peer review) is the author/funder. All rights reserved. No reuse allowed without permission.

\section{Supplementary Figure 3}

A

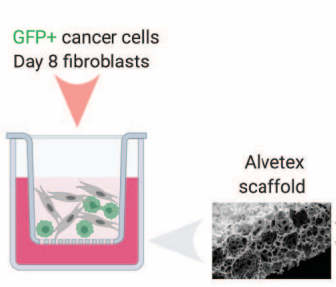

C
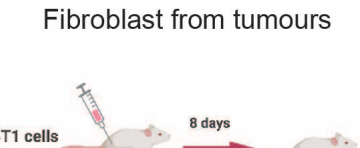

D

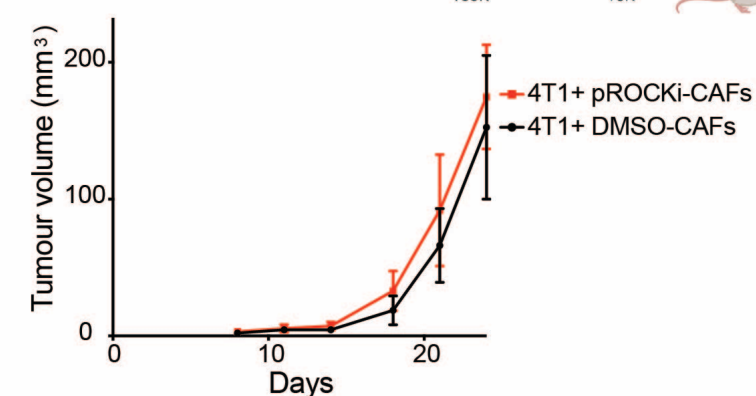

B
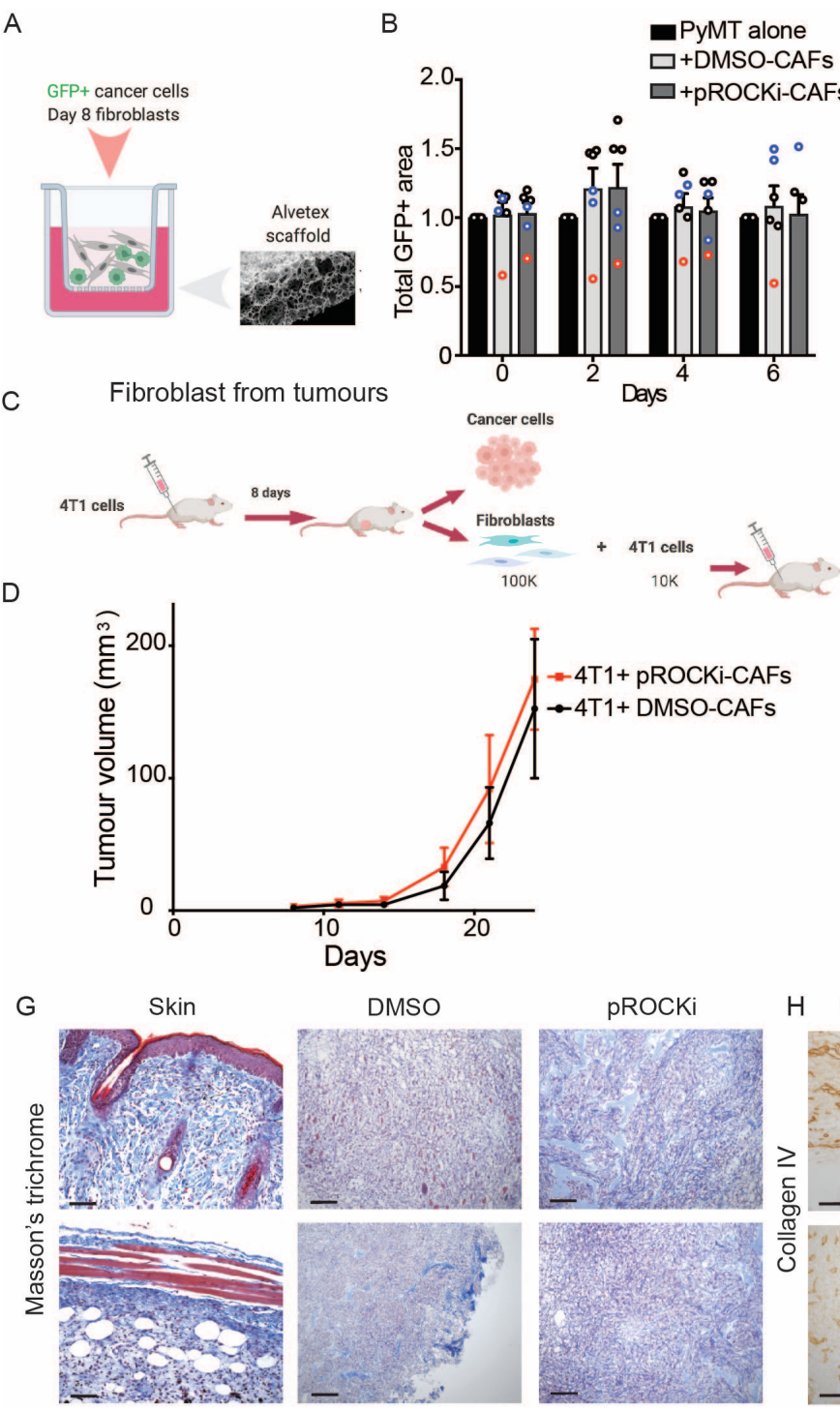

DMSO

pROCKi
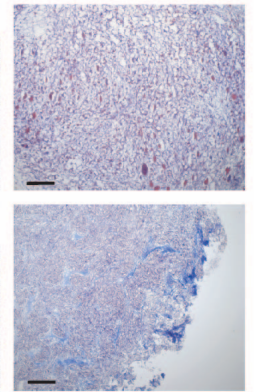

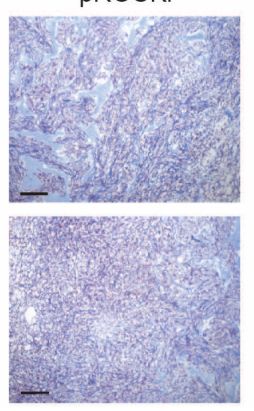

H Mammary gland

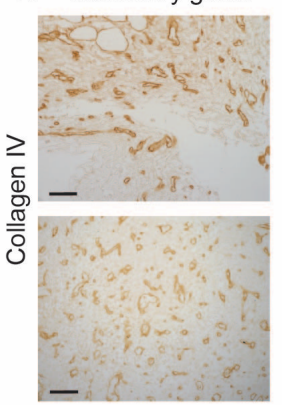

Fibroblast activation in vitro
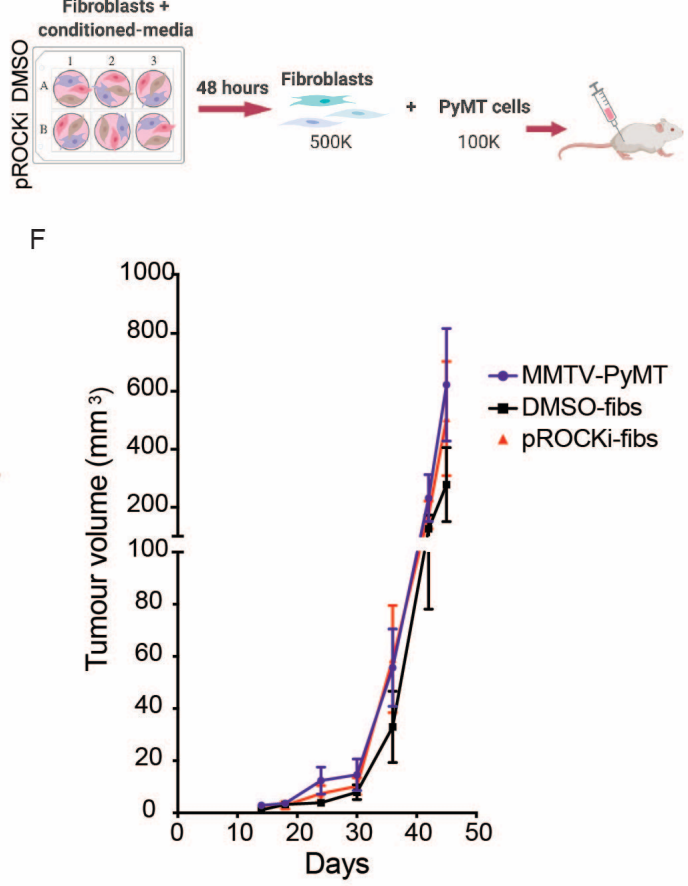

DMSO

pROCKi
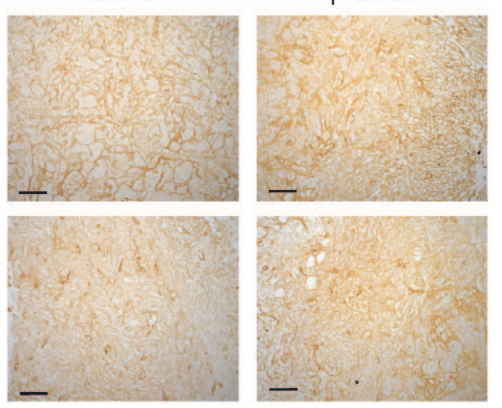
bioRxiv preprint doi: https://doi.org/10.1101/2021.06 15.448466; this version posted June 15, 2021. The copyright holder for this preprint (which was not certified by peer review) is the author/funder. All rights reserved. No reuse allowed without permission.

\section{Supplementary Figure 4}

A

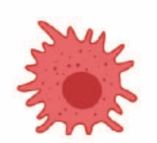

Pro -Tumorigenic macrophage markers
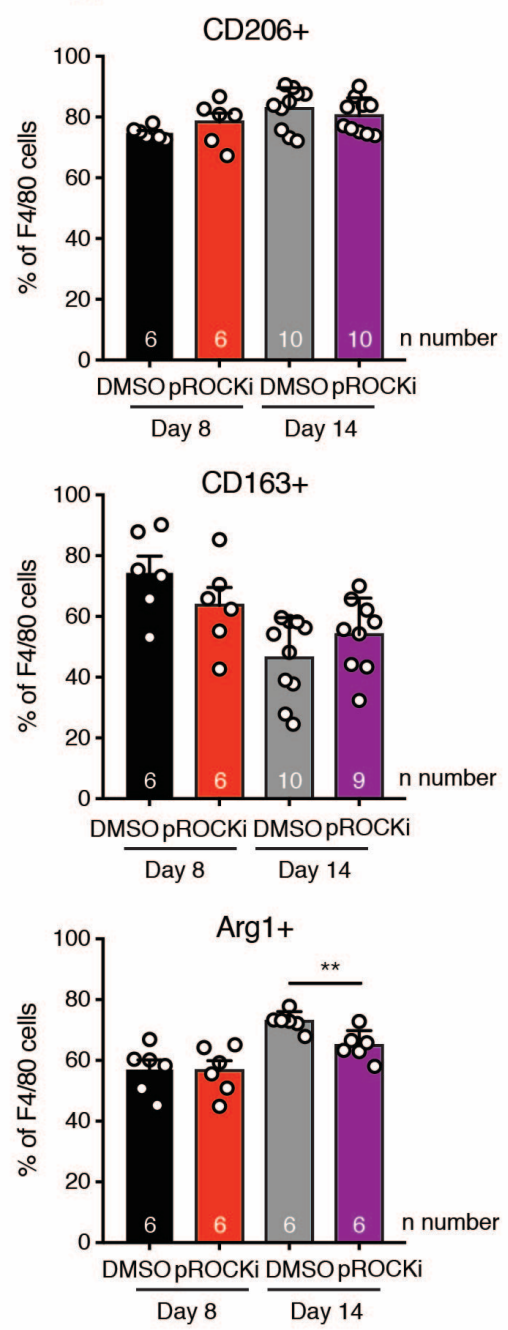

B
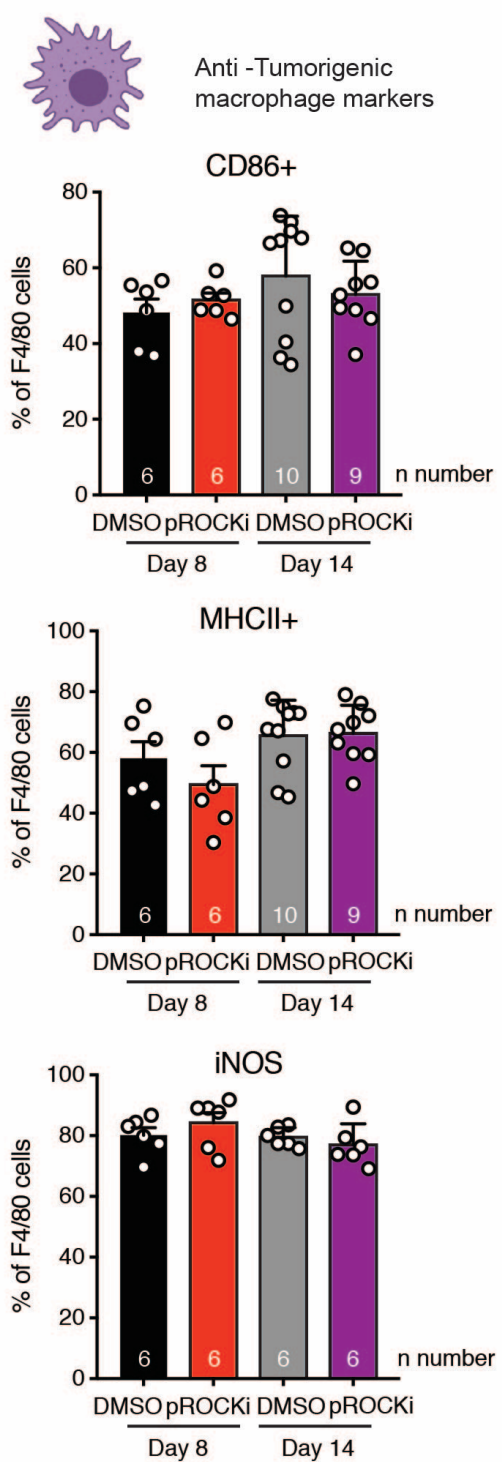

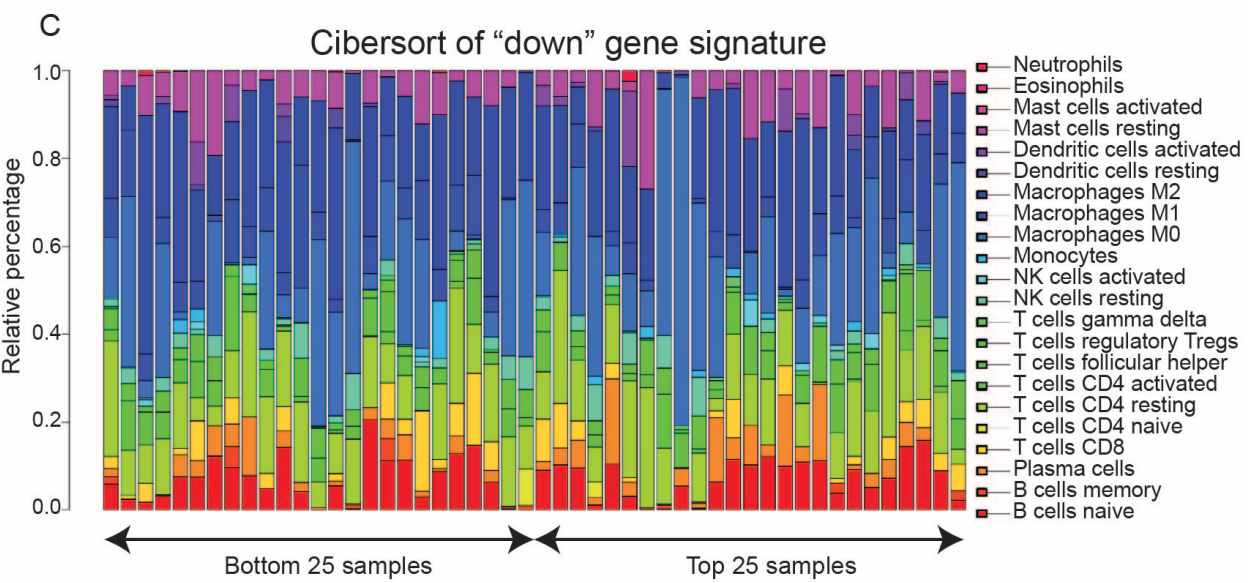


bioRxiv preprint doi: https://doi.org/10.1101/2021.06.15.448466; this version posted June 15, 2021. The copyright holder for this preprint (which was not certified by peer review) is the author/funder. All rights reserved. No reuse allowed without permission.

\section{Supplementary Figure 5}

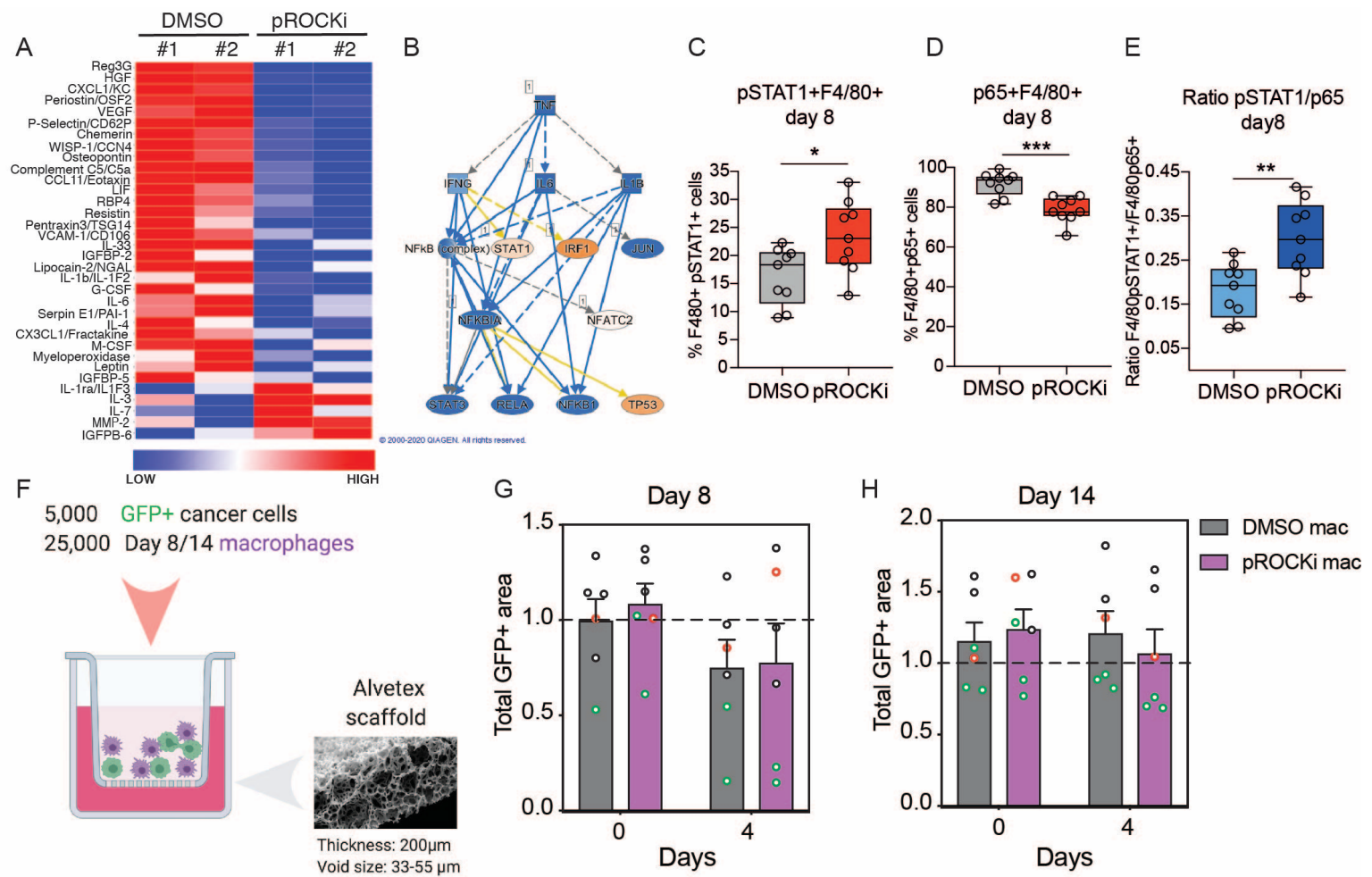

